\title{
Output Disturbance Observer Structure Applied to Run-to-Run Control for Semiconductor Manufacturing
}

\author{
An-Chen Lee, Yi-Ren Pan, and Ming-Tsung Hsieh
}

\begin{abstract}
Among the run-to-run (RtR) controllers, the EWMA controller, the double EWMA controller, and the predictor corrector controller are widely adopted in the semiconductor industry. This paper presents a unified framework for these controllers, which is called the output disturbance observer (ODOB) structure. The advantages of applying the ODOB structure are that the process output will be forced to the process target and the actual plant to the nominal plant while the process disturbance and the measurement noise are rejected. The relations of the tuned parameters of the above-mentioned controllers and the ODOB controller are discussed. Furthermore, based on the unified framework, the RtR controller with model mismatch and metrology delay can be analyzed systematically. In this paper, we analyze the robust stable conditions for the ODOB controller taking the model mismatch and metrology delay into consideration and provide a method to tune the controller.
\end{abstract}

Index Terms-Disturbance observer, double EWMA, EWMA, metrology delay, output disturbance observer (ODOB) controller, run-to-run controller.

\section{INTRODUCTION}

$\mathbf{R}$ ECENTLY, run-to-run (RtR) control technology has been widely used in the semiconductor manufacturing (e.g., [1]-[16]). Process disturbances such as shift disturbances caused by environmental change and regular maintenance, and drift disturbance by long-run operation are often produced in the processes. Analyzing the characteristic outputs of the previous runs, the RtR controller continuously modifies the model and then updates the input recipe for the next run to reduce variations between the process output and the target. Owing to the simplicity and robustness, the EWMA controller has been the most commonly used RtR controller in semiconductor manufacturing. Box and Jenkins [17] introduced the EWMA statistic, a minimum mean square error controller for the process disturbance following an $\operatorname{IMA}(1,1)$ time series process. Sachs et al. [3] proposed the RtR control scheme based on the EWMA statistic. They used the linear static

Manuscript received August 11, 2008; revised July 24, 2009; accepted September 20, 2010. Date of publication October 21, 2010; date of current version February 4, 2011. This work was supported by the National Science Council of the Republic of China, under Contract NSC 97-2221-E-009-070MY2.

The authors are with the Department of Mechanical Engineering, National Chiao-Tung University, Hsinchu 300, Taiwan (e-mail: aclee@mail. nctu.edu.tw; keen0405.me90g@nctu.edu.tw; u0036400@cc.ncu.edu.tw).

Color versions of one or more of the figures in this paper are available online at http://ieeexplore.ieee.org.

Digital Object Identifier 10.1109/TSM.2010.2088990 models to a design feedback-based RtR controller and used the EWMA statistic as an estimate of the process. Sachs et al. [3] successfully applied the EWMA controller to the RtR control, the use of the EWMA controller has been shown effectively in many processes including chemical mechanical polishing [4], chemical deposition [7], and plasma etching [8]. However, if there exists severe tool aging or the process drifts, the EWMA controller could not compensate for the steady drifts but produce an offset in the process output. For overcoming the offset produced by the EWMA controller, a predictor corrector controller (PCC) [2] and a double EWMA controller [11] were developed. Actually, the EWMA and the PCC controllers can be represented in terms of the internal model control (IMC) structure. Bulter and Stefani [2] used the IMC structure to design the PCC controller and Adivikolanu and Zafiriou [10] applied IMC structure to extend the EWMA controller. In addition to the EWMA, the double EWMA and the PCC controllers, several RtR control algorithms were proposed in the literature. Del Castillo and Yeh [6] used recursive least squares (RLS) algorithm to estimate the disturbances and update the process gain after each run. Wang and Mahajan [5] proposed an artificial neural network (ANN) model-based controller. The needs of copious parameters for constructing suitable networks and selecting training data are the drawbacks of ANN. Moreover, different approaches are proposed to find out APC optimal weights in many researches. [2] obtained the optimal weights by a searching method without considering the stability of the APC. [9] and [16] searched the weights of double EWMA by minimizing a performance tradeoff between long-run and transient responses, and [11] discussed the properties of PCC and double EWMA and found the weights by exhaustive searching. In this paper, given that the modeling uncertainty in terms of the additive uncertainty of the process model as opposed to the plant/model ratio, we provide a systematic method for obtaining the optimal parameters which guarantee the optimal nominal performance under the robust stability.

Concerning system stability issue, Ingolfsson and Sachs [1] studied the conditions for the stability of the EWMA controller and showed the allowable stable range against model mismatch. [9] derived the stable conditions for the double EWMA controller, but the metrology delay was not considered. Tseng et al. [12] derived the stable region of a MIMO EWMA controller. In [22], the single-tuned-parameter 


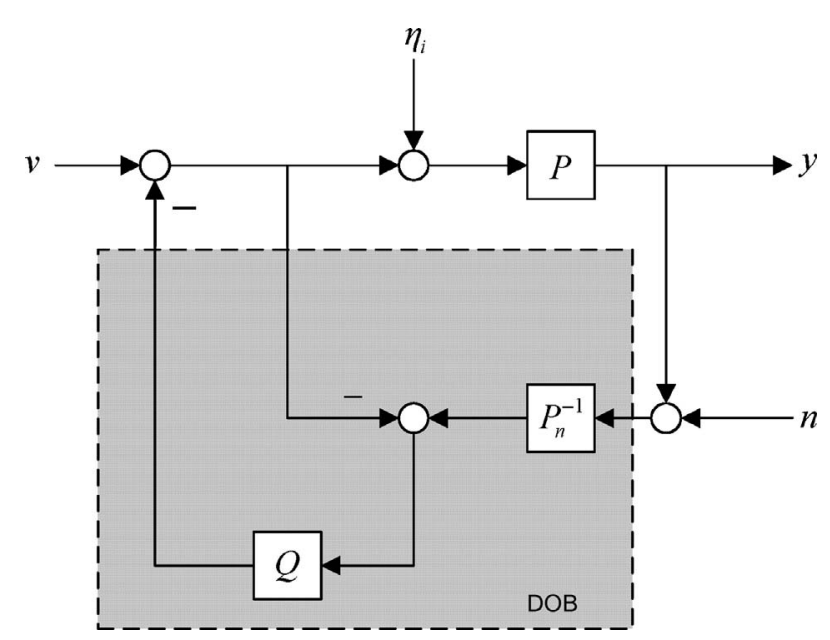

Fig. 1. Block diagram of the disturbance observer.

RLS of the double EWMA controller is applied to derive the stable conditions under special assumption, so it could not become general. Because metrology delay is very common in the implementation of APC in semiconductor manufacturing systems, it is very important to understand the effects of metrology delay on performance and stability of APC systems. In [10] and [13]-[15], the researchers discussed the system stability or performance of APC with metrology delay. Adivikolanu [10] derived the stable region of one run delay and only provided sufficient condition for large delay, and Wang et al. [13] simplified the double-EWMA by one tuned parameter to investigate the effect of metrology delay on performance without discussing stability condition. Good and Qin [14] investigated the effect of metrology delay on the closed-loop stability of a MIMO EWMA controller. However, mapping the stable conditions by the bilinear transform is quite complicated. Wu et al. [15] discussed the influence of metrology delay on both the transient and asymptotic properties of the product quality; the stability condition was not discussed neither. This paper provides an efficient and systematic augmented state-space method for obtaining stable region with/without finite metrology delays.

The disturbance observer (DOB) structure which reduces sensitivity to modeling error and enhances disturbance rejection properties is widely used in the field of motion control. The DOB is often applied to the inner-loop of the motion control structure to reject equivalent disturbance as much as possible for achieving precise position tracking. It was first introduced by Ohnishi [18] and refined by Umeno and Hori [19]. The block diagram of the DOB structure proposed by Umeno and Hori [19] is shown in Fig. 1, where $y$ is the output, $n$ the measurement noise, $P$ the plant, $P_{n}$ the nominal plant, $v$ the control input, $\eta_{i}$ the input disturbance, and $Q$ a lowpass filter. When the DOB structure is applied to a system, the system is forced to behave like its nominal plant, and the input disturbance and the measurement noise are rejected within the bandwidth of the Q-filter.

The input disturbance is always considered in the DOB structure in the past, since most physical systems suffer perturbations which are considered as input disturbances applied

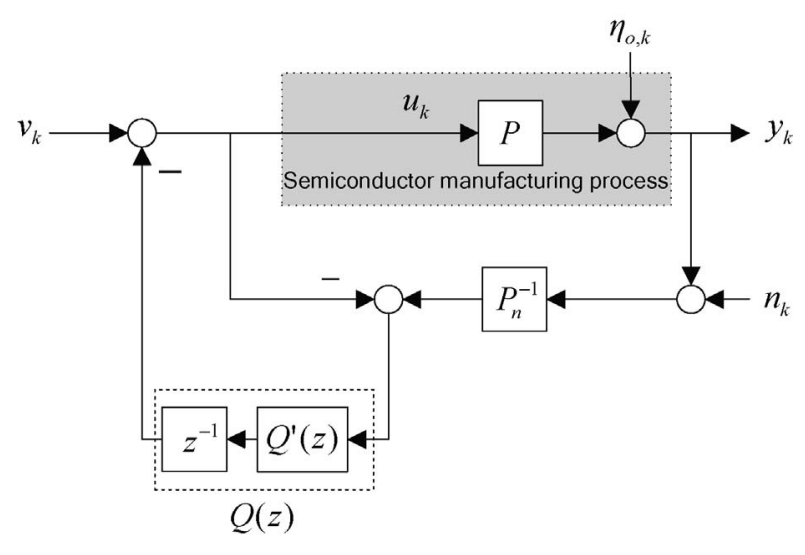

Fig. 2. Block diagram of RtR DOB structure.

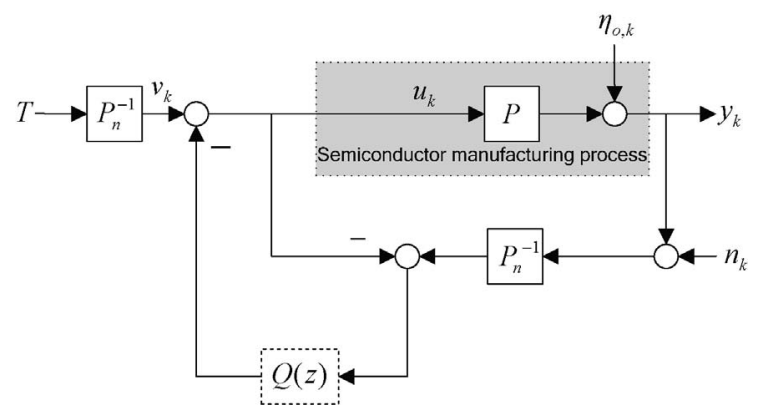

Fig. 3. Modified RtR DOB structure.

to the systems according to systems dynamics, such as friction force in motion control, eccentric force in rotor control, and heat loss in room-temperature control. Owing to the particular forms of production in semiconductor manufacturing, the process is usually established as a linear model including the process gain and the intercept term

$$
y_{k}=\eta_{o, k}+P \cdot u_{k}
$$

where $u_{k}$ is the process input (or input recipe), $y_{k}$ the process output, $\eta_{o, k}$ the intercept term, $P$ the process gain (or actual plant), and subscript $k$ means the batch index. Therefore, one modifies the original DOB into the RtR DOB structure shown in Fig. 2 where the intercept term is interpreted as the output disturbance and a unit delay operator $z^{-1}$ is considered in the feedback loop to be merged into the Q-filter due to the RtR control.

The DOB structure shown in Fig. 2 can force the actual plant to be equivalent to the nominal plant, i.e., the transfer function from control input $v_{k}$ to process output $y_{k}$ is $P_{n}$ within the bandwidth of the Q-filter. Based on this property, to make the transfer function from process target $T$ to process output $y_{k}$ to be unity, one must modify the block diagram of Fig. 2 by adding a pre-filter $P_{n}^{-1}$ with process target $T$ as input as shown in Fig. 3. The RtR DOB structure is further refined through the block diagram equivalence as shown in Fig. 4. This new structure is now called the output disturbance observer (ODOB) structure.

In this paper, the ODOB structure is used to design the RtR controller. When there are shift disturbances, drift distur- 


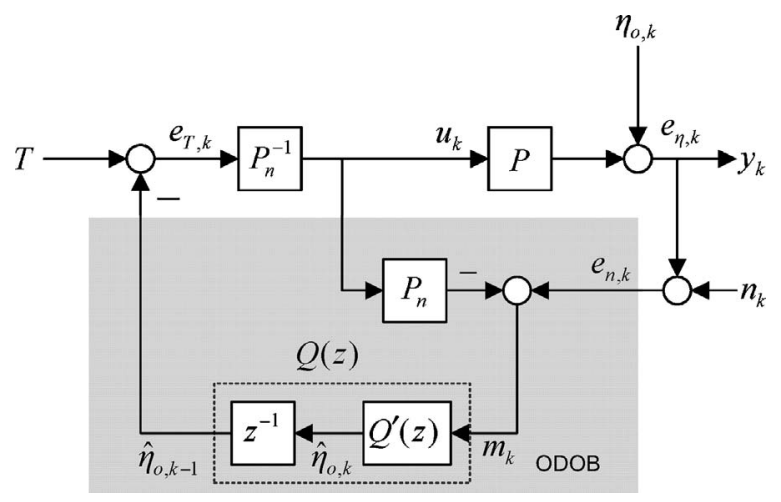

Fig. 4. Output disturbance observer (ODOB) structure.

bances, model errors or metrology delay in the processes, the observer can estimate the variation between the process output and the target of each run and generate the input recipe on-line to reduce the variation. The equivalence of the ODOB controller, EWMA controller, PCC controller, and double EWMA controller is illustrated through the relation between the tuned parameters. Thus, the ODOB structure provides the unified framework for these controllers, and consequently it can enhance insight into the well-known established algorithms, and contribute to an improved understanding of how these algorithms operate and why they can be used successfully in practical application.

The rest of this paper is organized as follows. Section II explains the advantage of the ODOB structure and applies it to the RtR controller. Besides, the relations of the tuned parameters among the EWMA, the double EWMA, the PCC and the ODOB controllers are analyzed. Section III analyzes the stable regions for the ODOB controller with or without metrology delay. Section IV provides a convenient and effective method to design the optimal tuned parameters of the ODOB controller for robust performance. In Section V, two examples with a third-order Q-filter are provided to show the performance improvement over existing controllers and the final section draws the conclusion.

\section{ODOB STRUCTURE APPLIED TO RTR CONTROL}

\section{A. ODOB Structure}

In the ODOB structure shown in Fig. 4, a unit delay operator is merged into the Q-filter in the feedback loop which is due to the RtR process characteristics, and this unit delay is different to any additional metrology delays. According to Fig. 4, the transfer functions from external inputs $\left[\begin{array}{ccc}T & \eta_{o, k} & n_{k}\end{array}\right]^{T}$ to internal states $\left[\begin{array}{lll}e_{T, k} & e_{\eta, k} & e_{n, k}\end{array}\right]^{T}$ can be represented as

$$
\begin{gathered}
{\left[\begin{array}{c}
e_{T, k} \\
e_{\eta, k} \\
e_{n, k}
\end{array}\right]=\frac{P_{n}}{P_{n}+\left(P-P_{n}\right) Q}\left[\begin{array}{ccc}
1 & -Q & -Q \\
P_{n}^{-1} P & (1-Q) & -P_{n}^{-1} P Q \\
P_{n}^{-1} P & (1-Q) & (1-Q)
\end{array}\right]} \\
{\left[\begin{array}{c}
T \\
\eta_{o, k} \\
n_{k}
\end{array}\right] .}
\end{gathered}
$$

Because all the nine transfer functions in (2) need to be stable to satisfy the internal stability [20] for the internal stabilizing conditions, one obtains that $P_{n}, P,\left[P_{n}+\left(P-P_{n}\right) Q\right]^{-1}$ (the characteristic equation from input $T$ to output $y$ ) and $Q$ should all be stable. In other words, the internal stability needs the input-output stability (I/O stability) and Q-filter stability, since the nominal plant and actual plant are constant gains in process control. The closed-loop transfer functions in the z-domain are obtained as follows:

$$
\begin{aligned}
Y(z)= & \frac{P}{P_{n}+\left(P-P_{n}\right) Q} \cdot T(z)+\frac{P_{n}(1-Q)}{P_{n}+\left(P-P_{n}\right) Q} \cdot H_{o}(z) \\
& -\frac{P Q}{P_{n}+\left(P-P_{n}\right) Q} \cdot N(z) \\
z^{-1} \hat{H}_{o}(z)= & \frac{Q\left(P-P_{n}\right)}{P_{n}+\left(P-P_{n}\right) Q} \cdot T(z)+\frac{Q P_{n}}{P_{n}+\left(P-P_{n}\right) Q} \cdot \mathrm{H}_{o}(z) \\
& +\frac{Q P_{n}}{P_{n}+\left(P-P_{n}\right) Q} \cdot N(z) .
\end{aligned}
$$

Let the $\mathrm{Q}$-filter be designed as $Q(z) \approx 1$ in the low frequency range, the transfer function of (3) becomes $Y(z) \approx$ $T(z)-N(z)$. If the measurement noise is ignored, the process output $y_{k}$ is equivalent to the process target $T$, i.e., the transfer function from $T$ to $y_{k}$ is 1 . Furthermore, in (4), if the actual plant $P$ is identical to the nominal plant $P_{n}$, the output of Q-filter $\hat{\eta}_{o, k-1}$ is equivalent to the output disturbance $\eta_{o, k}$, i.e., $\hat{\eta}_{o, k-1}$ is an estimator of $\eta_{o, k}$. Therefore, the ODOB structure provides the function of disturbance rejection. On the other hand, if the measurement noise exists, the Q-filter is designed as $Q(z) \approx 0$ in the high frequency range to filter it out. Hence, the ODOB structure provides the function of noise rejection.

\section{B. Particular Q-Filter Structure for APC}

The Butterworth low-pass filter is recommended in the Q-filter design by Umeno and Hori [19]; however, the filter can only suppress the general disturbance, but not completely eliminate the particular disturbances. Since the process shifts and drifts are often produced in semiconductor manufacturing because of the change of the environment or the long-run operation, the ODOB controller needs to compensate for process shifts and drifts for zero steady state error in the process output. Therefore, we present the particular Q-filter structure in the z-domain as follows.

The first-order Q-filter with a unit inherent delay operator is designed as

$$
Q(z)=\frac{b_{1}}{z+a_{1}}
$$

i.e., $Q^{\prime}(z)=\frac{b_{1} z}{z+a_{1}}$ and $a_{1}, b_{1}$ are the tuned parameters. The Q-filter needs to have unit DC gain since the requirement of $Q(z) \approx 1$ in the low frequency range. Therefore, the tuned parameters $a_{1}$ and $b_{1}$ have the constraint of

$$
b_{1}=1+a_{1} .
$$

To check whether the designed Q-filter can compensate for the process disturbances, including shift and drift disturbances, one can apply the final value theorem to the transfer function from the process disturbance to the process output in (3). Let the model mismatch $\xi$ be defined as $\xi=\frac{P}{P_{n}}$, then the first-order Q-filter is substituted into (3) with $T=0$. The final values of 
the process output with the constraint of (6) for shift and drift disturbances are obtained, respectively.

1) Final value of the process output for shift disturbance

$$
\begin{aligned}
\left(H_{o}(z)=\right. & \left.\frac{z}{z-1}\right) \\
& \lim _{z \rightarrow 1}\left(1-z^{-1}\right) \frac{(1-Q)}{1+(\xi-1) Q} \cdot H_{o}=0 .
\end{aligned}
$$

2) Final value of the process output for drift disturbance

$$
\begin{aligned}
& \left(H_{o}(z)=\frac{\delta z}{(z-1)^{2}}, \delta: \text { drift slope }\right) \\
& \quad \lim _{z \rightarrow 1}\left(1-z^{-1}\right) \frac{(1-Q)}{1+(\xi-1) Q} \cdot H_{O}=\frac{\delta}{\xi\left(1+a_{1}\right)} .
\end{aligned}
$$

From (7) and (8), the first-order Q-filter can compensate for the shift disturbance, but with an offset in the process output for the drift disturbance. Because the first-order Q-filter cannot compensate for the drift disturbance, the second-order Q-filter with a unit delay operator is introduced as follows:

$$
\left.Q(z)=\frac{b_{1} z+b_{2}}{z^{2}+a_{1} z+a_{2}} \quad \text { (i.e., } Q^{\prime}(z)=\frac{b_{1} z^{2}+b_{2} z}{z^{2}+a_{1} z+a_{2}}\right)
$$

where $a_{1}, a_{2}, b_{1}$, and $b_{2}$ are the tuned parameters. Since the Q-filter also needs to have unit DC gain, the tuned parameters have the constraint of

$$
b_{1}+b_{2}=1+a_{1}+a_{2} .
$$

According to the final value theorem, if the second-order Q-filter can compensate for the shift and drift disturbances, the tuned parameters need to satisfy the two constraints of

$$
\left\{\begin{array}{l}
b_{1}=a_{1}+2 \\
b_{2}=a_{2}-1 .
\end{array}\right.
$$

It seems that there exist three constraints for the second-order Q-filter. However, adding two constraints in (11) results in the constraint of (10), which means the second-order Q-filter has only two independent parameters.

\section{EWMA, Double EWMA, and PCC Controllers Represented in the $O D O B$ Structure}

The EWMA, double EWMA, and PCC controllers are reviewed in Appendix A. The EWMA controller represented in terms of the ODOB structure is illustrated as follows. Let $P=\beta$ and $P_{n}=b, m_{k}=y_{k}-P_{n} \cdot u_{k}$ (under noise-free assumption), $\hat{\eta}_{o, k-1}$ is the estimated disturbance by the Q-filter and $u_{k}=e_{T, k} \cdot P_{n}^{-1}=\frac{T-\hat{\eta}_{o, k-1}}{P_{n}}$, i.e., the recipe $u_{k}$ for the $k$ run is $\frac{T-\hat{\eta}_{o, k-1}}{P_{n}}$. The function of the EWMA filter $F_{E}(z)$ in (A4) associated with a unit inherent delay operator is the same as the first-order Q-filter. Therefore, the EWMA controller can be equivalently represented in the ODOB structure. Similarly, the double EWMA controller and the PCC controller can also be equivalently represented in the second-order ODOB structure, where the double EWMA filter $F_{D}(z)$ obtained from (A9) and the PCC filter $F_{P}(z)$ obtained from (A13), each associated with a unit inherent delay. Thus, the ODOB structure provides a unified framework for EWMA, double EWMA and PCC controllers and one can use the unified framework to design the RtR controller with high order Q-filter.

Because the EWMA, double EWMA, and PCC controllers can be equivalently represented in the ODOB structure, the following relation between the first-order Q-filter and the EWMA controller is obtained:

$$
a_{1}=\omega-1 .
$$

Similarly, the following relations between the second-order Qfilter and the double EWMA controller are obtained:

$$
\begin{gathered}
a_{1}=-2+\omega_{1}+\omega_{2} \\
a_{2}=1-\omega_{1}
\end{gathered}
$$

and the relations of the PCC controller are

$$
\begin{gathered}
a_{1}=-2+\omega_{1}^{*}+\omega_{2}^{*} \\
a_{2}=1-\omega_{1}^{*}-\omega_{2}^{*}+\omega_{1}^{*} \omega_{2}^{*} .
\end{gathered}
$$

In fact, the final value theorem can be applied in the same way to prove that the EWMA controller can compensate only for a shift disturbance and will have an offset in the process output for drift disturbance, but the double EWMA and the PCC controllers can compensate for both shift and drift disturbances.

\section{STABILITY OF THE ODOB CONTROLLER}

\section{A. Stable Conditions of the ODOB Controller Without Metrology Delay}

Recalling the internal stabilizing conditions needs the I/O stability and Q-filter stability. In terms of model mismatch, $\xi=\frac{P}{P_{n}}$, the $\mathrm{I} / \mathrm{O}$ stability condition, $\left[P_{n}+\left(P-P_{n}\right) Q\right]^{-1}$ can be rewritten as $[1+(\xi-1) Q]^{-1} P_{n}^{-1}$ and further simplified as $[1+(\xi-1) Q]^{-1}$. Obviously, the model mismatch $\xi$ will influence the pole locations of $[1+(\xi-1) Q]^{-1}$ and thus the I/O stability. Furthermore, the overall stable region of the ODOB is the intersection of the $\mathrm{I} / \mathrm{O}$ stable region and the $\mathrm{Q}$-filter stable region.

To obtain the stable region of the Q-filter, a criterion for determining the stability is the Jury's test [21]. For the firstorder Q-filter in (5), the stable constraint using the Jury's test is

$$
-1<a_{1}<1 .
$$

Similarly, for the second-order Q-filter in (9), the stable constraints are

$$
\begin{gathered}
1+a_{1}+a_{2}>0 \\
1-a_{1}+a_{2}>0 \\
\left|a_{2}\right|<1 .
\end{gathered}
$$

From system stability viewpoint, the weightings of the EWMA, double EWMA, and PCC controllers can be set greater than 1 as long as the EWMA filter in (A4), double EWMA filter in (A9), and PCC filter in (A13) satisfy the stability constraints.

To analyze the I/O stability, one applies the augmented state-space method and directly utilizes the Jury's test for 


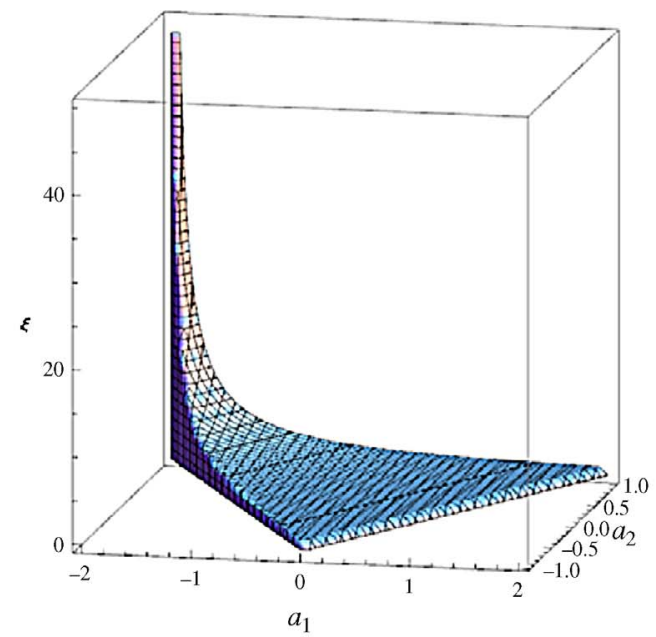

(a)
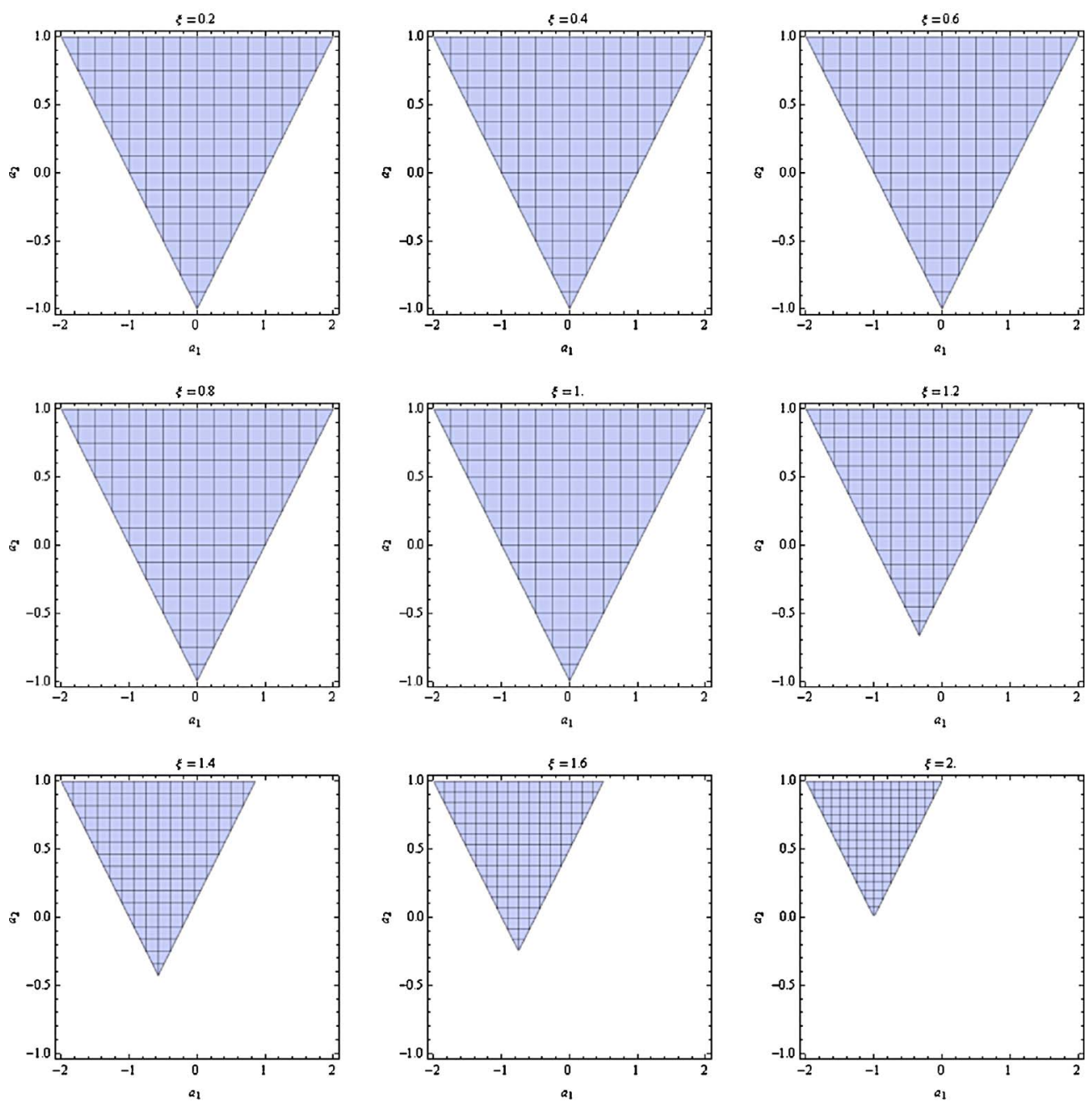

(b)

Fig. 5. (a) 3-D stable region of the ODOB with a second-order Q-filter and no metrology delay. (b) Cross-sections for different model mismatches in (a). 
the systems with/without metrology delay and the method is more effective and systematic that it can be extended easily to derive the stable conditions for the MIMO system. In Fig. 4, $\hat{\eta}_{o}$ and $m$ are the state variables of the ODOB structure and the measurement noise is ignored. The process target $T$ can be set to zero without loss of generality for stability analysis. Then, the output equation of the ODOB structure is represented as

$$
y_{k}=-P P_{n}^{-1} \cdot \hat{\eta}_{o, k-1}+\eta_{o, k} .
$$

The output $y_{k}$ is simply the linear combination of the state variable $\hat{\eta}_{o, k}$ and the disturbance. In the following, one presents the details for the second-order filter and only shows the results of the first-order filter. Let the second-order Q-filter in (9) be represented in the controllable canonical form as follows:

$$
\begin{gathered}
{\left[\begin{array}{l}
x_{1, k+1} \\
x_{2, k+1}
\end{array}\right]=\left[\begin{array}{cc}
0 & 1 \\
-a_{2} & -a_{1}
\end{array}\right]\left[\begin{array}{l}
x_{1, k} \\
x_{2, k}
\end{array}\right]+\left[\begin{array}{l}
0 \\
1
\end{array}\right] m_{k}} \\
\hat{\eta}_{o, k-1}=\left[\begin{array}{ll}
b_{2} & b_{1}
\end{array}\right]\left[\begin{array}{l}
x_{1, k} \\
x_{2, k}
\end{array}\right] .
\end{gathered}
$$

Define the state vector $\mathbf{Z}_{k}=\left[\begin{array}{lll}x_{1, k} & x_{2, k} & \hat{\eta}_{o, k-2}\end{array}\right]^{T}$, then (22) and (23) can be combined as

$$
\mathbf{Z}_{k+1}=\mathbf{A} \cdot \mathbf{Z}_{k}+\mathbf{B} \cdot m_{k}
$$

where $\mathbf{A}=\left[\begin{array}{ccc}0 & 1 & 0 \\ -a_{2} & -a_{1} & 0 \\ b_{2} & b_{1} & 0\end{array}\right]$, and $\mathbf{B}=\left[\begin{array}{l}0 \\ 1 \\ 0\end{array}\right]$.

In Fig. $4, m_{k}=\left(1-P P_{n}^{-1}\right) \cdot \hat{\eta}_{o, k-1}+\eta_{o, k}$, and can be rewritten as

$$
m_{k}=\mathbf{G} \cdot \mathbf{Z}_{k+1}+\boldsymbol{\eta}_{o, k}
$$

where $\mathbf{G}=\left[\begin{array}{lll}0 & 0 & (1-\xi)\end{array}\right]$. Then, (25) is substituted into (24) to obtain the dynamic equation of the ODOB structure, or

$$
\mathbf{Z}_{k+1}=\overline{\mathbf{A}} \cdot \mathbf{Z}_{k}+\overline{\mathbf{B}} \cdot \eta_{o, k}
$$

where $\overline{\mathbf{A}}=(\mathbf{I}-\mathbf{B} \cdot \mathbf{G})^{-1} \cdot \mathbf{A}$ and $\overline{\mathbf{B}}=(\mathbf{I}-\mathbf{B} \cdot \mathbf{G})^{-1} \cdot \mathbf{B}$. The system is $\mathrm{I} / \mathrm{O}$ stable if the eigenvalues of the transition matrix $\overline{\mathbf{A}}$ are all inside the unit circle. According to the Jury's test, the I/O stable region with the constraints of (11) is

$$
0<\xi<\frac{4}{a_{1}-a_{2}+3} .
$$

In addition, the overall stable region is the intersection of stable regions of Q-filter and (27). For the ODOB with a firstorder Q-filter and without metrology delay, the stable region is the intersection of $0<\xi<\frac{2}{1+a_{1}}$ and (17). Fig. 5(a) shows the 3-D stable region of the ODOB with a second-order Qfilter and no metrology delay, and Fig. 5(b) shows the crosssections for different model mismatches $\xi$ to assist in visual interpretation.

\section{B. Stable Regions of the $O D O B$ Controller with Metrology Delay}

As mentioned in the introduction, metrology delay is a very important issue in RtR process control because it will influence not only the output performance but the system stability. The block diagram of the ODOB structure with metrology delay

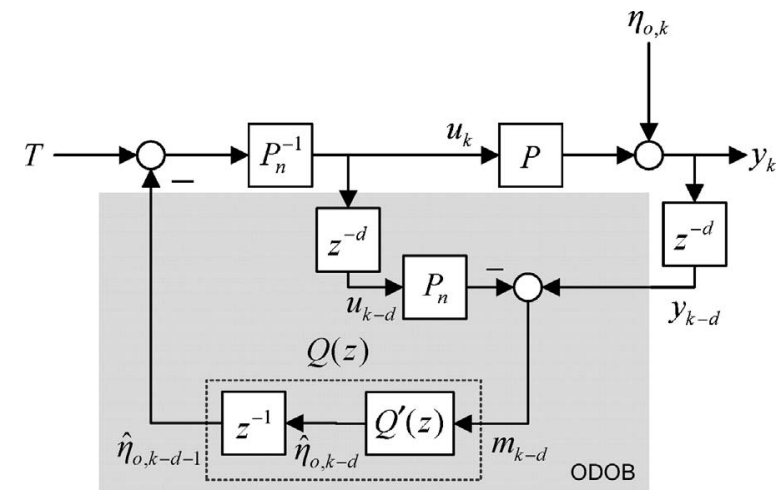

Fig. 6. ODOB structure with metrology delay.

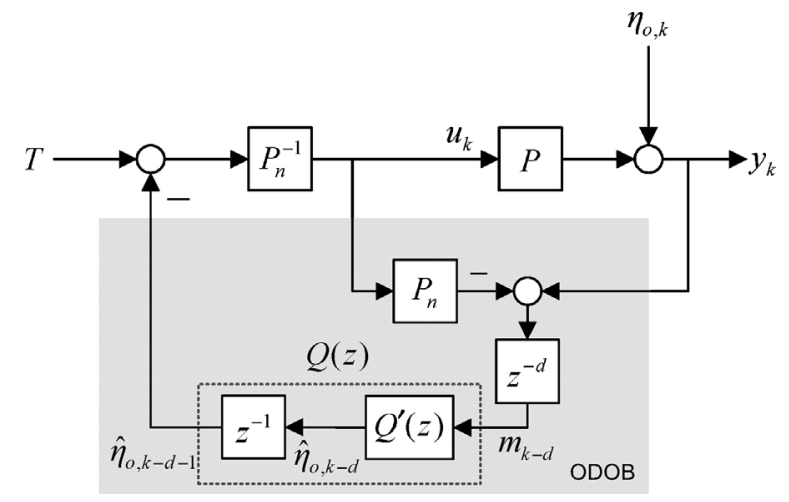

Fig. 7. Modified ODOB structure with metrology delay.

is shown in Fig. 6, where $d$ is the additional metrology delay number. A time delay operator $z^{-d}$ is added in the ODOB structure to match the run series and $m_{k-d}=y_{k-d}-P_{n} \cdot \mathbf{u}_{k-d}$. The ODOB structure with metrology delay can be further modified through the block diagram equivalence as shown in Fig. 7. Considering the delay problem with compensation for the shift and drift disturbances, one can obtain the constraints for the second-order Q-filter as

$$
\left\{\begin{array}{l}
b_{1}=a_{1}+2+d\left(a_{1}+a_{2}+1\right) \\
b_{2}=a_{2}-1-d\left(a_{1}+a_{2}+1\right) .
\end{array}\right.
$$

In Fig. 7, the output equation of the ODOB structure with metrology delay is

$$
y_{k}=-P P_{n}^{-1} \cdot \hat{\eta}_{o, k-d-1}+\eta_{o, k} .
$$

The second-order Q-filter is again represented in the controllable canonical form as (22) and (23). In Fig. 7, $M_{k-d}$ can be rewritten as

$$
m_{k-d}=\mathbf{G} \cdot \mathbf{Z}_{k+1-2 d}+\eta_{o, k-d} .
$$

Equation (30) is substituted into (24) and the state equation of the ODOB with metrology delay is

$$
\mathbf{Z}_{k+1-d}=\mathbf{A} \cdot \mathbf{Z}_{k-d}+\mathbf{B} \cdot \mathbf{G} \cdot \mathbf{Z}_{k+1-2 d}+\mathbf{B} \cdot \eta_{o, k-d} .
$$

In the following, the metrology delays of one and two runs are discussed, respectively. 
1) Delay of One Run: Let $d=1$, and then the state equation (31) of the ODOB with delay of one run is obtained

$$
\mathbf{Z}_{k}=(\mathbf{A}+\mathbf{B} \cdot \mathbf{G}) \cdot \mathbf{Z}_{k-1}+\mathbf{B} \cdot \eta_{o, k-1} .
$$

Define the state vector $\overline{\mathbf{Z}}_{k}=\left[\begin{array}{ll}\mathbf{Z}_{k-1}^{T} & \eta_{o, k-1}\end{array}\right]^{T}$, the state equation (32) can be written as

$$
\overline{\mathbf{Z}}_{k+1}=\overline{\mathbf{A}}_{1} \cdot \overline{\mathbf{Z}}_{k}+\overline{\mathbf{B}}_{1} \cdot \eta_{o, k}
$$

where $\overline{\mathbf{A}}_{1}=\left[\begin{array}{c|c}\mathbf{A}+\mathbf{B} \cdot \mathbf{G} & \mathbf{B} \\ \hline \mathbf{0} & \mathbf{0}\end{array}\right]=\left[\begin{array}{ccc|c}0 & 1 & 0 & 0 \\ -a_{2} & -a_{1} & (1-\xi) & 1 \\ b_{2} & b_{1} & 0 & 0 \\ 0 & 0 & 0 & 0\end{array}\right]$, and $\overline{\mathbf{B}}_{1}=\left[\begin{array}{c}0 \\ 0 \\ 0 \\ 1\end{array}\right]$.

According to the Jury's stability test, the I/O stable region with one run delay is obtained in

$$
\frac{4\left(a_{1}+1\right)}{\left(3 a_{1}+a_{2}+5\right)}<\xi<\frac{4 a_{1}-a_{2}+a_{1}^{2}+5}{\left(a_{1}+2\right)^{2}} .
$$

Fig. 8(a) shows the stereographic stable region and Fig. 8(b) shows the cross-sections of Fig. 8(a) for different model mismatches $\xi$. The overall stable region of the process with one run delay is the intersection of (34) and (18)-(20). For the ODOB with a first-order Q-filter, the overall stable region with delay of one run is the intersection of $0<\xi<\frac{2+a_{1}}{1+a_{1}}$ and (17).

2) Delay of Two Runs: Let $d=2$, and then the state equation (31) with delay of two runs is obtained

$$
\mathbf{Z}_{k-1}=\mathbf{A} \cdot \mathbf{Z}_{k-2}+\mathbf{B} \cdot \mathbf{G} \cdot \mathbf{Z}_{k-3}+\mathbf{B} \cdot \eta_{o, k-2} .
$$

Define the state vector $\tilde{\mathbf{M}}_{k}=\left[\begin{array}{llll}\mathbf{Z}_{k-2}^{T} & \mathbf{Z}_{k-3}^{T} & \eta_{o, k-2} & \eta_{o, k-1}\end{array}\right]^{T}$, (35) can be written as

$$
\tilde{\mathbf{M}}_{k+1}=\overline{\mathbf{A}}_{2} \cdot \tilde{\mathbf{M}}_{k}+\overline{\mathbf{B}}_{2} \cdot \eta_{o, k}
$$

where

$$
\overline{\mathbf{A}}_{2}=\left[\begin{array}{c|c|c}
\mathbf{F} & \mathbf{J} & \mathbf{0} \\
\hline \mathbf{0} & \mathbf{0} & \mathbf{I} \\
\hline \mathbf{0} & \mathbf{0} & \mathbf{0}
\end{array}\right]
$$

$$
=\left[\begin{array}{cccccc|c|c}
0 & 1 & 0 & 0 & 0 & 0 & 0 & 0 \\
-a_{2} & -a_{1} & 0 & 0 & 0 & (1-\xi) & 1 & 0 \\
b_{2} & b_{1} & 0 & 0 & 0 & 0 & 0 & 0 \\
1 & 0 & 0 & 0 & 0 & 0 & 0 & 0 \\
0 & 1 & 0 & 0 & 0 & 0 & 0 & 0 \\
0 & 0 & 1 & 0 & 0 & 0 & 0 & 0 \\
\hline 0 & 0 & 0 & 0 & 0 & 0 & 0 & 1 \\
\hline 0 & 0 & 0 & 0 & 0 & 0 & 0 & 0
\end{array}\right], \overline{\mathbf{B}}_{2}=\left[\begin{array}{c}
0 \\
0 \\
0 \\
0 \\
0 \\
0 \\
0 \\
1
\end{array}\right],
$$

$$
\mathbf{F}=\left[\begin{array}{cc}
\mathbf{A} & \mathbf{B} \cdot \mathbf{G} \\
\mathbf{I} & \mathbf{0}
\end{array}\right], \quad \mathbf{J}=\left[\begin{array}{l}
\mathbf{B} \\
\mathbf{0}
\end{array}\right]
$$

and the stable region is obtained as

$$
\begin{aligned}
& 18 a_{1}^{3}+\left(25 a_{2}+78\right) a_{1}^{2}+\left(70 a_{2}+12 a_{2}^{2}+114\right) a_{1} \\
& \frac{+56+2 a_{2}^{3}+16 a_{2}^{2}+50 a_{2}-\sqrt{\theta}}{2\left[8 a_{1}^{3}+\left(36+12 a_{2}\right) a_{1}^{2}+\left(54+6 a_{2}^{2}+36 a_{2}\right) a_{1}+\left(a_{2}+3\right)^{3}\right]} \\
& <\xi<\frac{4\left(a_{1}+a_{2}+2\right)}{5 a_{1}+3 a_{2}+7}
\end{aligned}
$$

where

$$
\begin{aligned}
\theta= & 4 a_{1}^{6}+\left(24+4 a_{2}\right) a_{1}^{5}+\left(60+a_{2}^{2}+4 a_{2}\right) a_{1}^{4} \\
& +\left(112-12 a_{2}^{2}-60 a_{2}\right) a_{1}^{3}-\left(4 a_{2}^{3}-204+76 a_{2}^{2}+164 a_{2}\right) a_{1}^{2} \\
& -\left(128 a_{2}^{2}+16 a_{2}^{3}+128 a_{2}-240\right) a_{1} \\
& +\left(112-16 a_{2}-16 a_{2}^{3}-64 a_{2}^{2}\right) .
\end{aligned}
$$

Also, the overall stable region is the intersection of (37) and (18)-(20). Fig. 9(a) shows the stable region of the ODOB with a second-order Q-filter and delay of two runs, and Fig. 9(b) shows the cross-sections for different model mismatches $\xi$. For the ODOB with a first-order Q-filter, the overall stable region with delay of two runs is the intersection of $0<\xi<$ $\frac{2+3 a_{1}+\sqrt{a_{1}^{2}+4}}{2\left(1+a_{1}\right)}$ and (17).

From Figs. 5(b), 8(b), and 9(b), it is apparent that the stable region is getting smaller when model mismatch $\xi$ departures from 1 in some specific metrology delay. Furthermore, by overlapping these three figures, we observed that when there is no model mismatch, $\xi=1$, the metrology delay does not affect the stable region, but when there is a model mismatch, larger metrology delay does not necessarily yield smaller stable region. For example, see $\xi=1.6$ in different delays. It is also interesting that the stable region for lager delays is not necessarily included in the one for smaller delays. Therefore, to make the stability of the system less affected by the metrology delay, e.g., from 0 to 2, we should find Q-filter parameters within these intersection regions.

\section{Optimal Nominal PeRformance Under the ROBUST STABILITY}

\section{A. Robust Stability of the ODOB Controller}

As discussed in the above section, the stability of the ODOB controller is influenced by the model mismatch, the Q-filter, and metrology delay. In addition to the stability problem, the existence of the model mismatch and metrology delay brings about poor performance of the processes. The model mismatch exists because the experimental data are insufficient to establish the process model. Actually, the process always changes with time and the modeling uncertainty will be presented due to the use of the same model for the process. Therefore, the available process model is not always accurate, that is, the actual plant $P$ is not equivalent to the nominal plant $P_{n}$ in the process. The model mismatch $\xi$ has been defined as $\xi=\frac{P}{P_{n}}$ in the past. Here the relation of the actual plant $P$ and the nominal plant $P_{n}$ is defined as

$$
P=P_{n}+\Delta
$$

where $\Delta$ is the modeling uncertainty (or estimated standard errors) in terms of the additive uncertainty of the process model. One can calculate the estimated standard errors $\Delta$ 


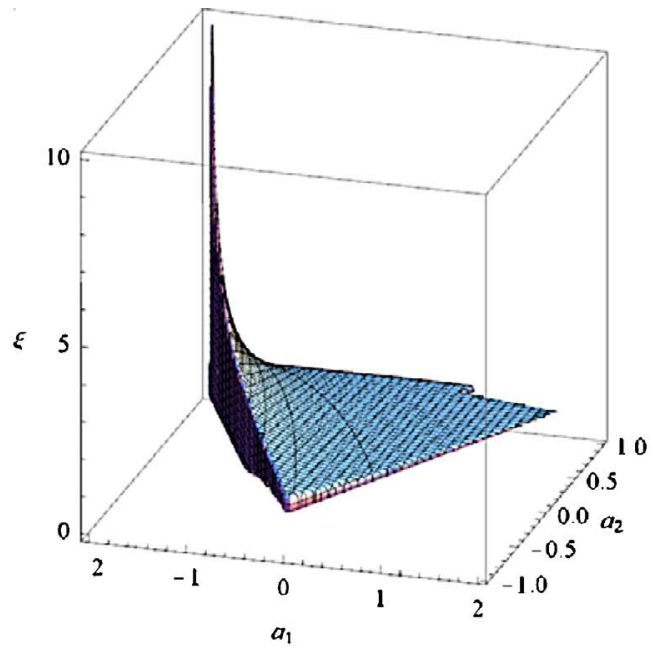

(a)
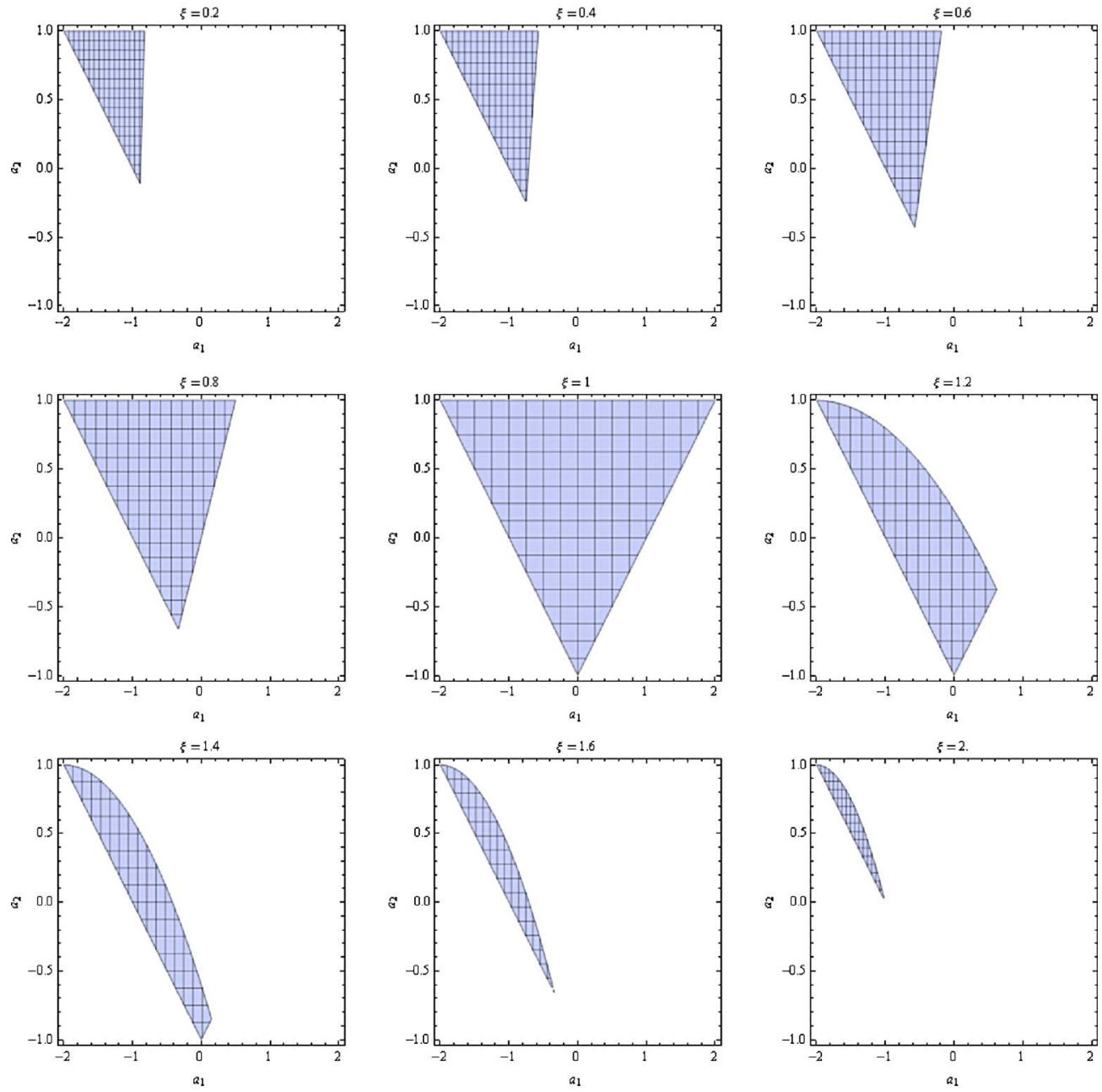

(b)

Fig. 8. (a) 3-D stable region of the ODOB with a second-order Q-filter and one metrology delay. (b) Cross-sections for different model mismatches in (a). 


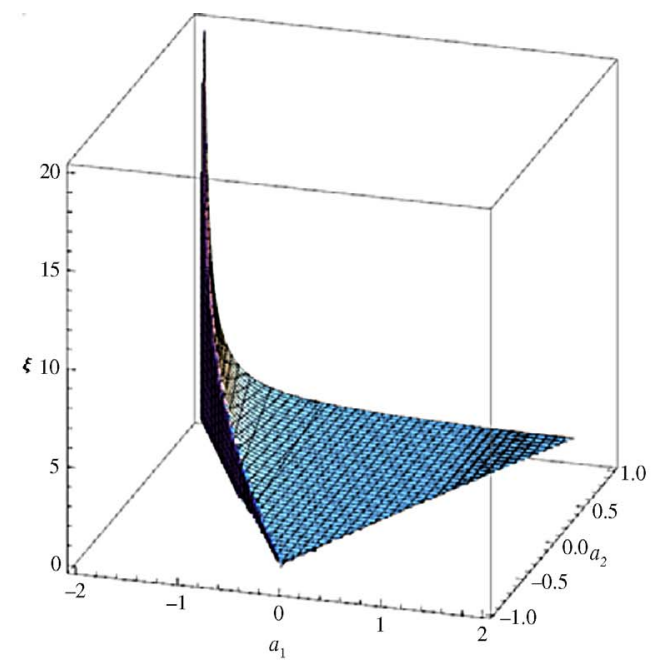

(a)
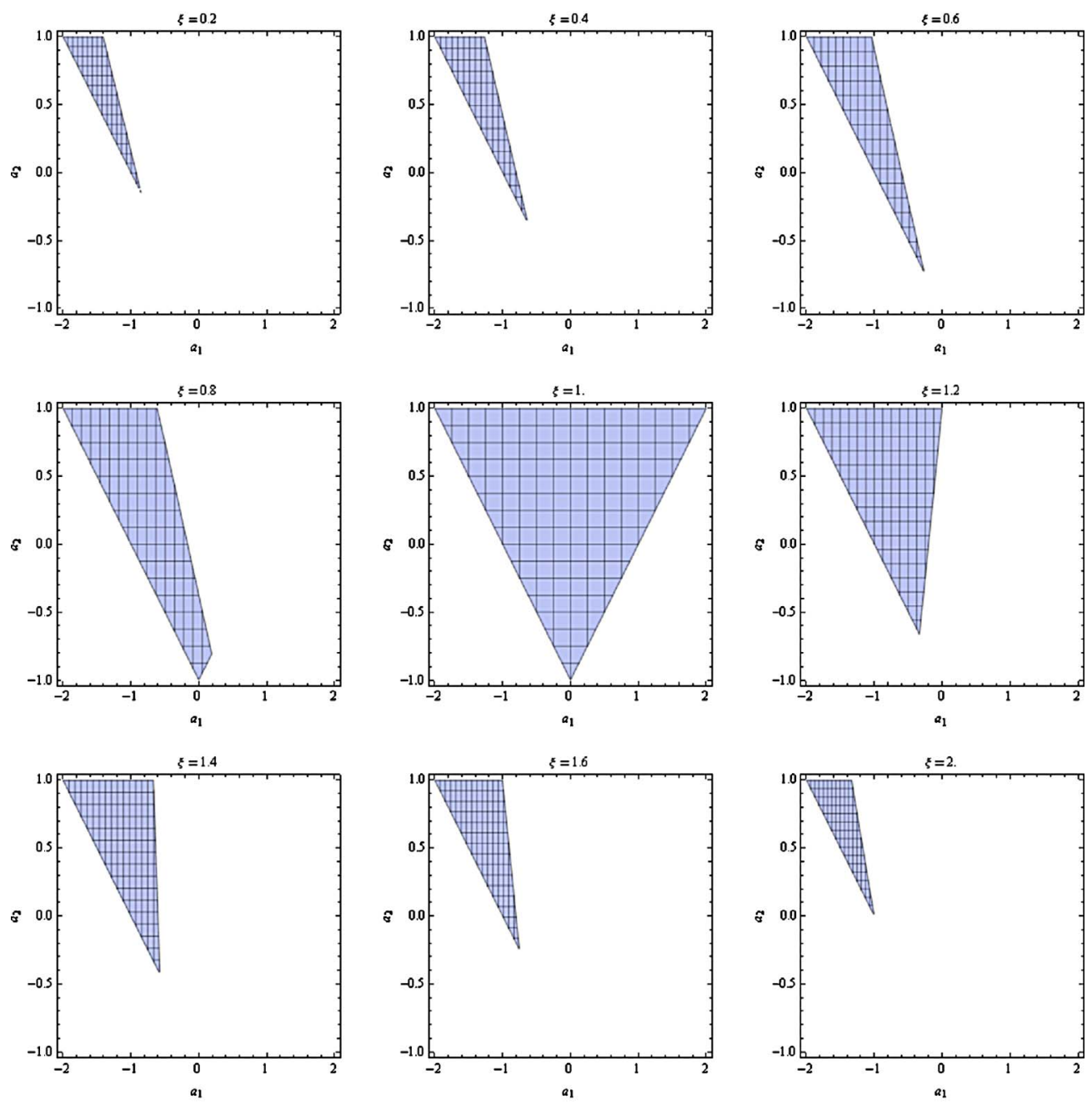

(b)

Fig. 9. (a) 3-D stable region of the ODOB with a second-order Q-filter and two metrology delays. (b) Cross-sections for different model mismatches in (a). 


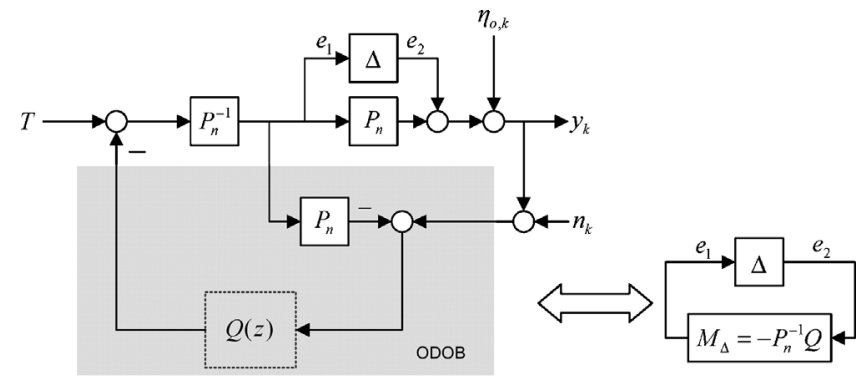

(a)

(b)

Fig. 10. (a) ODOB structure with additive modeling uncertainty. (b) Standard $\Delta-M_{\Delta}$ loop for robust stability analysis of ODOB structure.

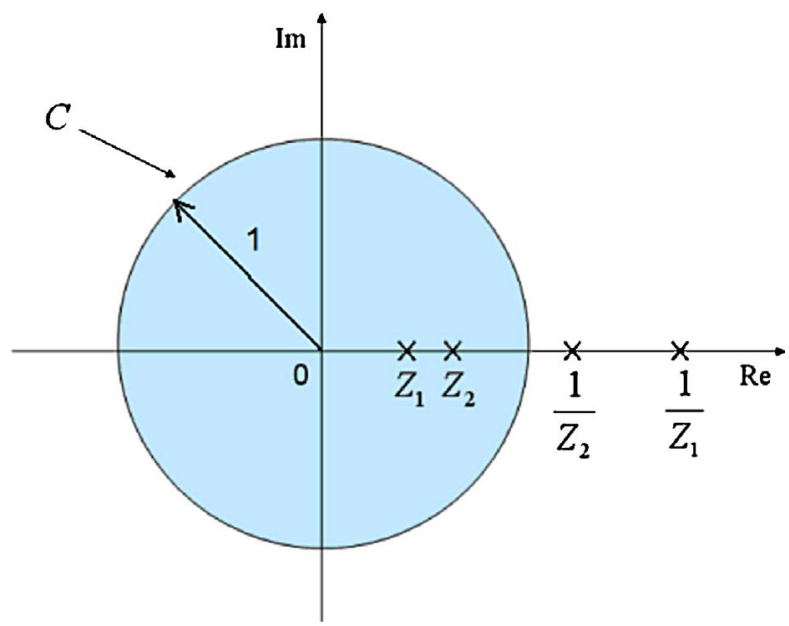

Fig. 11. Diagram showing contour $\mathrm{C}$ and poles $z=z_{1}, z=z_{2}, z=1 / z_{1}$, and $z=1 / z_{2}$.

from the historic data and the approach can be referred to Beck and Arnold [23]. The relation between the modeling uncertainty and the model mismatch is $|\Delta|=P_{n}|\xi-1|$. The ODOB structure with additive modeling uncertainty is shown in Fig. 10(a), which can be further merged into a standard $\Delta-M_{\Delta}$ loop of the small gain theorem [24] which is usually used to obtain robust stability in traditional robust control and shown in Fig. 10(b).

According to the small gain theorem, suppose $M_{\Delta} \in R H_{\infty}$, then the interconnected system is well-posed and internally stable for all $\Delta \in R H_{\infty}$ with

$$
\|\Delta\|_{\infty}\left\|M_{\Delta}\right\| \leq 1
$$

where $M_{\Delta}$ is the transfer function from $e_{1}$ to $e_{2}$ obtained as

$$
M_{\Delta}=-P_{n}^{-1} Q \text {. }
$$

The transfer function $M_{\Delta}$ is related to the nominal plant $P_{n}$ and the Q-filter. Therefore, according to the assumption of the small gain theorem $\left(M_{\Delta} \in R H_{\infty}\right)$, the second-order Q-filter with (18)-(20) needs to be stable since $P_{n}$ is just a constant gain.

If a bound of the possible modeling uncertainty $\Delta$ is known in advance for the process and the tuned parameters of the ODOB controller satisfy the small gain theorem in (39), the robust stability of ODOB controller can be realized.

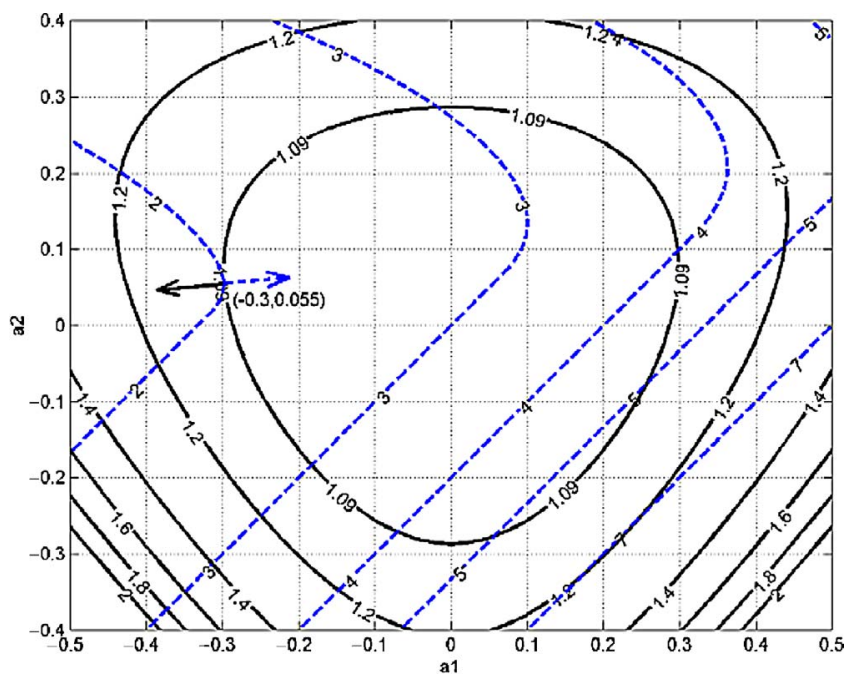

Fig. 12. Contours of SSE equation (black solid line) and $\|Q\|_{\infty}$ (blue dash line).

\section{B. Optimal Performance of the ODOB Controller Without Metrology Delay}

In the above part, we introduced robust stability and realized a robust ODOB controller by satisfying small gain theorem. In this part, one will introduce a method to tune the parameters of the ODOB controller which guarantee the optimal nominal performance under the robust stability for the RtR control.

If a boundary of the modeling uncertainty $\|\Delta\|_{\infty}=$ $\gamma$ is known in advance, the robust stable condition is $\left\|-P_{n}^{-1} Q\right\|_{\infty} \leq \frac{1}{\gamma}$ and can be rewritten as $\|Q\|_{\infty} \leq \frac{1}{\left|-P_{n}^{-1}\right| \cdot \gamma}$. On the other hand, if $\|Q\|_{\infty}=\varepsilon$ for any positive $\varepsilon$ is known in advance, the tolerance for the modeling uncertainty is $\|\Delta\|_{\infty} \leq \frac{1}{\left|-P_{n}^{-1}\right| \cdot \varepsilon}$. There are lots of combinations of the tuned parameters $a_{1}$ and $a_{2}$ which satisfy the robust stable constraints of the closed-loop system. To tune the optimal parameters to meet the performance, one applies the sum square error (SSE) of the process output as a performance index. The minimum SSE indicates the fast transient response. The optimal parameters with the minimum SSE can be solved immediately by the Parseval's theorem [25]. In Fig. 4, without considering the measurement noise, the error equation is obtained as

$$
\begin{aligned}
E(z)=Y(z)-T(z) & =\left[\frac{(\xi-1)(1-Q)}{1+(\xi-1) Q}\right] \cdot T(z) \\
& +\left[\frac{(1-Q)}{1+(\xi-1) Q}\right] \cdot H_{o}(z) .
\end{aligned}
$$

In (41), the process target $T$ can be set to zero without loss of generality. The performance for the condition with $P=P_{n}$ is used as the benchmark, and then the second-order Q-filter in (9) is substituted into (41). When the drift disturbance $H_{o}(z)=$ $\frac{\delta z}{(z-1)^{2}}$ is considered and (11) is applied, the error equation is obtained as

$$
E(z)=\frac{\delta z}{z^{2}+a_{1} z+a_{2}}=\frac{\delta z}{\left(z-z_{1}\right)\left(z-z_{2}\right)}
$$




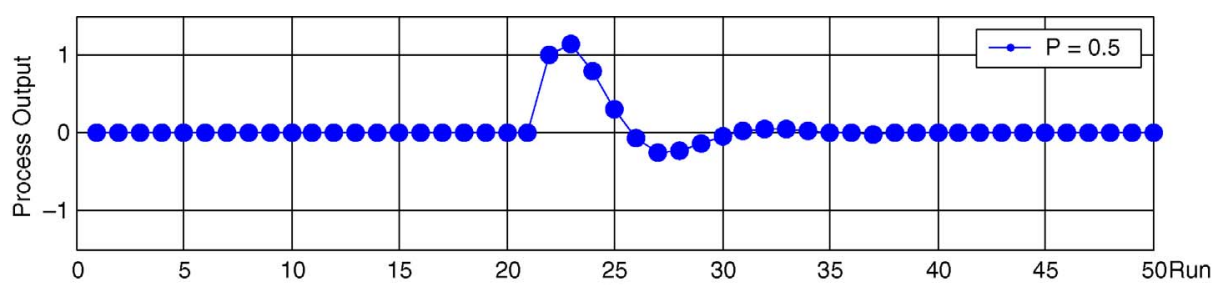

(a)

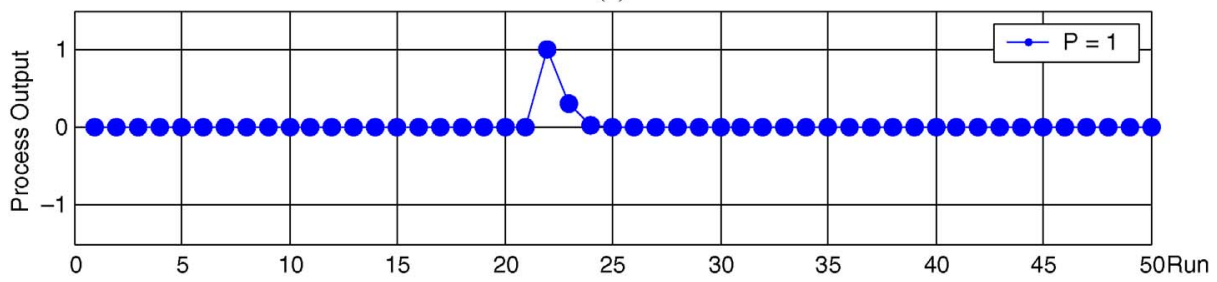

(b)

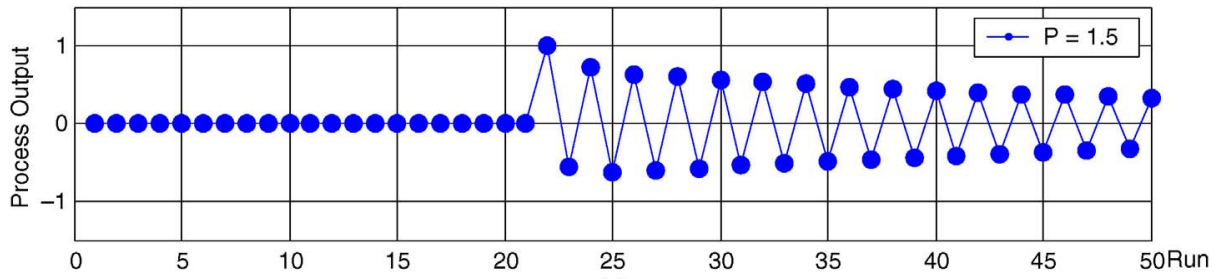

(c)

Fig. 13. Process outputs for drift disturbance $(\delta=1)$ occurring after run 20 with the parameters $a_{1}=-0.3$ and $a_{2}=0.055$. (a) $P_{n}=1$ and $P=0.5$. (b) $P_{n}=1$ and $P=1$. (c) $P_{n}=1$ and $P=1.5$.

where $z_{1}$ and $z_{2}$ are the closed-loop poles. According to Parseval's theorem, the SSE equation is derived as

$$
\sum_{k=0}^{\infty} e_{k}^{2}=\frac{1}{z_{1} z_{2}} \times \frac{1}{2 \pi j} \oint_{c} \frac{\delta^{2} z}{\left(z-z_{1}\right)\left(z-z_{2}\right)\left(z-\frac{1}{z_{1}}\right)\left(z-\frac{1}{z_{2}}\right)} d z
$$

where $\mathrm{C}$ is taken as the unit circle centered at the origin. Referring to Fig. 11, one finds that the poles at $z=z_{1}$ and $z=z_{2}$ are inside the contour $\mathrm{C}$, but the poles at $z=1 / z_{1}$ and $z=1 / z_{2}$ are outside the contour $\mathrm{C}$. Therefore, the residues at poles $z=z_{1}$ and $z=z_{2}$ can be easily evaluated and the SSE equation can be simplified as

$$
\sum_{k=0}^{\infty} e_{k}^{2}=\frac{-\delta^{2}\left(a_{2}+1\right)}{\left(a_{2}-1\right)\left(1+a_{2}-a_{1}\right)\left(1+a_{2}+a_{1}\right)} .
$$

The SSE equation obtained in (44) is related to the tuned parameters $a_{1}$ and $a_{2}$. Take the partial differentiation of the SSE equation with respect to $a_{1}$ and $a_{2}$, respectively

$$
\begin{aligned}
\frac{\partial \sum e^{2}}{\partial a_{1}} & =-\frac{2 \delta^{2} a_{1}\left(1+a_{2}\right)}{\left(a_{2}-1\right)\left(-1+a_{1}^{2}-2 a_{2}-a_{2}^{2}\right)^{2}} \\
\frac{\partial \sum e^{2}}{\partial a_{2}} & =\frac{2\left(-\delta^{2} a_{1}^{2}+\delta^{2} a_{2}+2 \delta^{2} a_{2}^{2}+\delta^{2} a_{2}^{3}\right)}{\left(a_{2}-1\right)^{2}\left(1-a_{1}^{2}+2 a_{2}+a_{2}^{2}\right)^{2}} .
\end{aligned}
$$

Equations (45) and (46) represent the sensitivity of the controller performance to the choice of the filter parameters $a_{1}$ and $a_{2}$. The larger the absolute values of (45) and (46) are, the more sensitive parameters $a_{1}$ and $a_{2}$ to the controller performance will be. For the minimum point of the SSE equation, the necessary conditions are $\frac{\partial \sum e^{2}}{\partial a_{1}}=0$ and $\frac{\partial \sum e^{2}}{\partial a_{2}}=$ 0 . That is, the numerators of the two equations are both zero. The optimal parameters of the second-order Q-filter in the

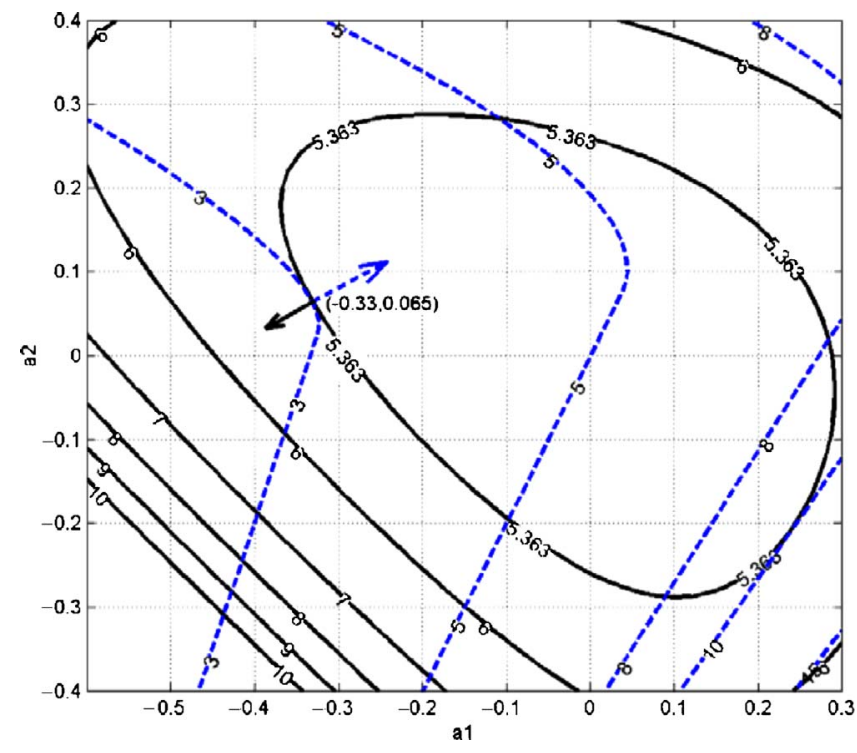

Fig. 14. Contours of SSE equation with delay of one run (black solid line) and $\|Q\|_{\infty}$ (blue dash line).

sense of minimum SSE for the benchmark (i.e., $P=P_{n}$ ) are obtained as $a_{1}=0$ and $a_{2}=0$, i.e., $Q=\frac{2 z-1}{z^{2}}\left(\|Q\|_{\infty}=3\right)$. The optimal parameters for the benchmark can guarantee the process output to be internally stable under the model uncertainty $\|\Delta\|_{\infty} \leq \frac{1}{3 \cdot\left|-P_{n}^{-1}\right|}$. If the model uncertainty is larger than $\frac{1}{3 \cdot\left|-P_{n}^{-1}\right|}$, appropriate parameters have to be found to ensure the robust stability. In the literature, the Lagrange multiplier method is applied to solve the constrained optimum design problem and here the constraint equation is $\|Q\|_{\infty}=\varepsilon$. The Lagrange function denoted as $L_{a}$ is defined by using SSE 


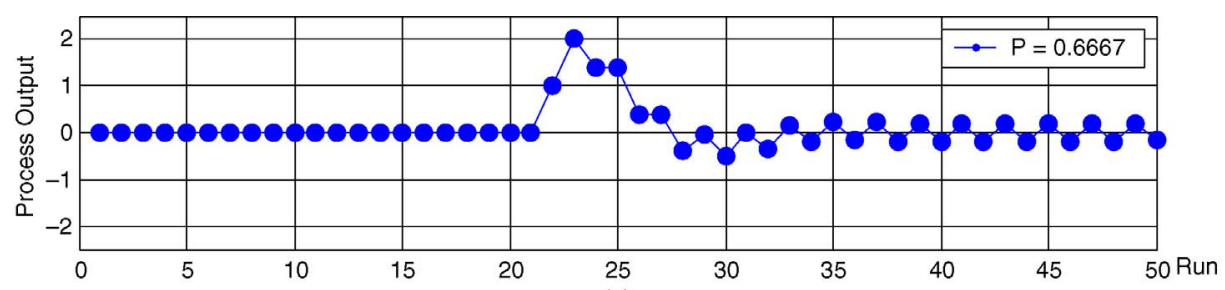

(a)

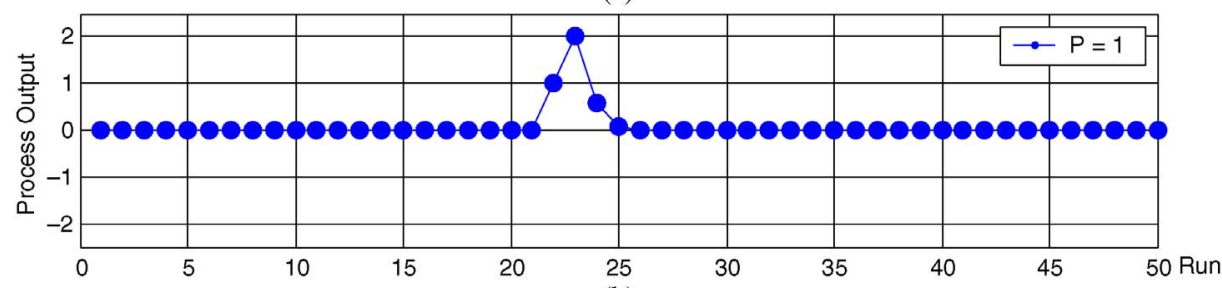

(b)

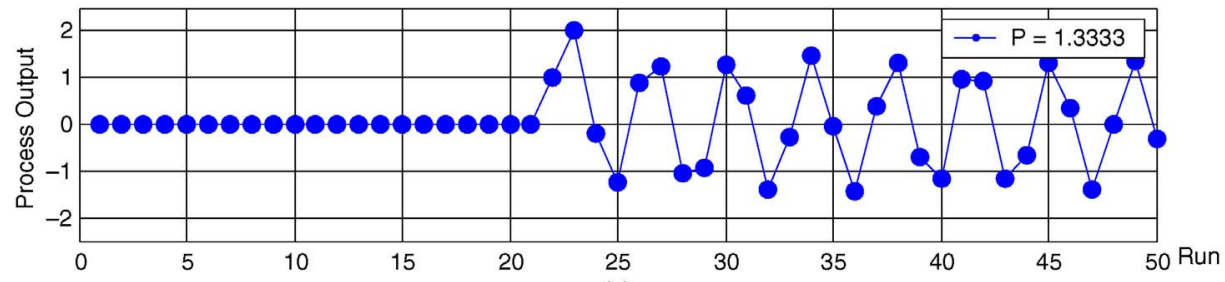

(c)

Fig. 15. Process outputs with delay of one run for drift disturbance $(\delta=1)$ occurring after run 20 and the parameters are set $a_{1}=-0.33$ and $a_{2}=0.065$. (a) $P_{n}=1$ and $P=0.6667$. (b) $P_{n}=1$ and $P=1$. (c) $P_{n}=1$ and $P=1.3333$.

and constraint equation as

$$
L_{a}=\sum e^{2}+\mu\left(\|Q\|_{\infty}\right) \quad\left(\|Q\|_{\infty}=\varepsilon ; \varepsilon>0\right)
$$

where $\mu$ is the Lagrange multiplier. The optimum points of the second-order Q-filter are located at $\mathbf{X}^{*}=\left(a_{1}^{*}, a_{2}^{*}\right)$ and $\nabla L_{a}\left(\mathbf{X}^{*}\right)=\nabla\left(\sum e^{2}\left(\mathbf{X}^{*}\right)\right)+\mu \cdot \nabla\left(\left\|Q\left(\mathbf{X}^{*}\right)\right\|_{\infty}\right)=0$ [26]. The gradient function $\nabla L_{a}\left(\mathbf{X}^{*}\right)=0$ infers that the angle between vector of $\nabla\left(\sum e^{2}\left(\mathbf{X}^{*}\right)\right)$ and vector of $\nabla\left(\left\|Q\left(\mathbf{X}^{*}\right)\right\|_{\infty}\right)$ must be $180^{\circ}$ (positive $\mu$ ) or $0^{\circ}$ (negative $\mu$ ). Since the $\|Q\|_{\infty}$ is difficult to obtain analytically, in the following, the design of the second-order ODOB is demonstrated using the geometric Lagrange multiplier method. Suppose the benchmark $P=P_{n}=$ 1 and the slope $\delta$ of drift disturbance is 1. Fig. 12 shows the contours of $\sum e^{2}\left(a_{1}, a_{2}\right)$ and $\left\|Q\left(a_{1}, a_{2}\right)\right\|_{\infty}$, respectively. According to previous discussion, the optimum parameters for the benchmark are $a_{1}=0$ and $a_{2}=0$, i.e., $Q=\frac{2 z-1}{z^{2}}\left(\|Q\|_{\infty}=\right.$ 3, $\left.\|\Delta\|_{\infty} \leq \frac{1}{3}\right)$ and $\sum e^{2}(0,0)=1$. If the tolerance of model uncertainty increases to $\frac{1}{2}$, i.e., $\|\Delta\|_{\infty} \leq \frac{1}{2}$ and $\|Q\|_{\infty}=2$, the optimum solution can be solved as $\mathbf{X}^{*}=\left(a_{1}^{*}, a_{2}^{*}\right)=$ $(-0.3,0.055)$, i.e., $\|Q(z)\|_{\infty}=\left\|\frac{1.7 z-0.945}{z^{2}-0.3 z+0.055}\right\|_{\infty}=2$ and minimum SSE $\sum e^{2}(-0.3,0.055)=1.09$ as illustrated in Fig. 12 . Furthermore, the angle between $\nabla\left\|Q\left(\mathbf{X}^{*}\right)\right\|_{\infty}$ (the blue dash arrow) and $\nabla \sum e^{2}\left(\mathbf{X}^{*}\right)$ (the black solid arrow) is $180^{\circ}$.

It corresponds to the double EWMA controller with $\omega_{1}=$ 0.945 and $\omega_{2}=0.755$. While mapping the ODOB into the PCC controller one obtained $\omega_{1}^{*}=0.85 \pm 0.1803 i$ and $\omega_{2}^{*}=0.85 \mp$ $0.1803 i$, but the complex weightings are never used in PCC controller. In summary, it can be shown that the parameter set of the second-order ODOB is larger than that of the PCC and is equivalent to that of the double EWMA. Fig. 13 shows the simulation of the process outputs where the nominal plant is

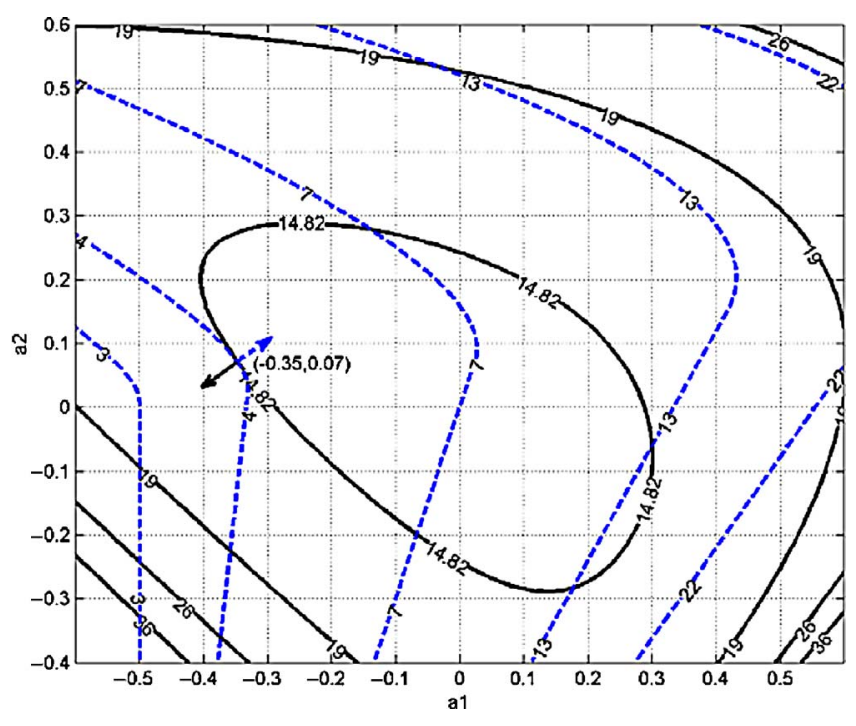

Fig. 16. Contours of SSE equation with delay of two runs (black solid line) and $\|Q\|_{\infty}$ (blue dash line).

set $P_{n}=1$ and the actual plants are $P=0.5, P=1$, and $P=1.5$, respectively, corresponding to $\|\Delta\|_{\infty} \leq \frac{1}{2}$, and the drift disturbance $(\delta=1)$ occurs after run 20, and the SSE $\sum e^{2}$ of the benchmark $\left(P=P_{n}=1\right)$ is 1.09 .

\section{Optimal Performance of the ODOB Controller with Metrology Delay}

When the additional metrology delay is considered, the optimal parameters of the second-order Q-filter for the benchmark $\left(P=P_{n}\right)$ can be obtained from the following lemma. 


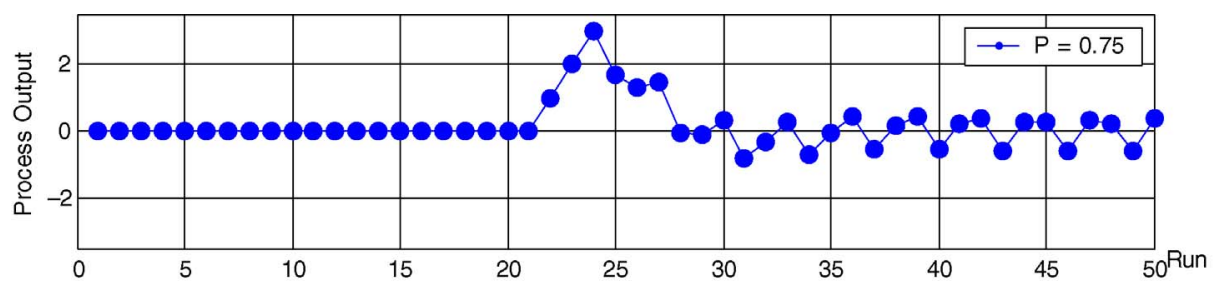

(a)

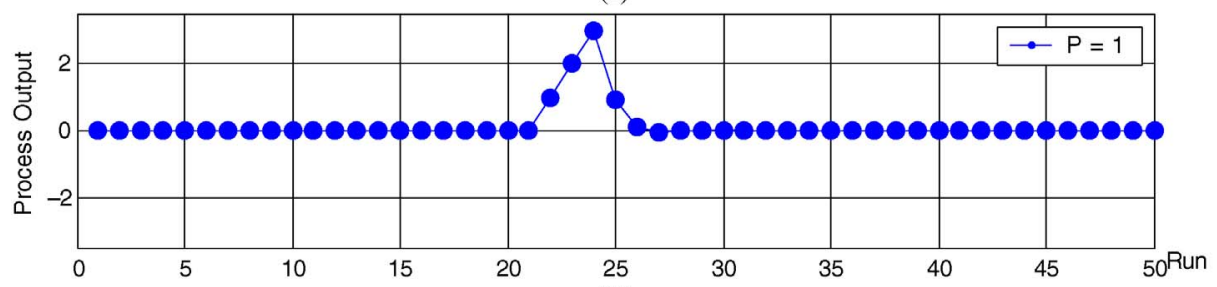

(b)

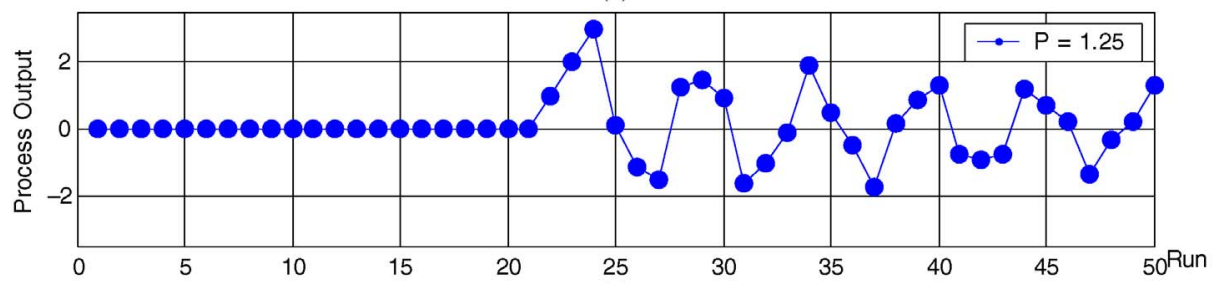

(c)

Fig. 17. Process outputs with delay of two runs for drift disturbance $(\delta=1)$ occurring after run 20 and the parameters are set $a_{1}=-0.35$ and $a_{2}=0.07$. (a) $P_{n}=1$ and $P=0.75$. (b) $P_{n}=1$ and $P=1$. (c) $P_{n}=1$ and $P=1.25$.

Lemma 1: The optimal parameters of the second-order Q-filter with metrology delay for the benchmark are obtained as $a_{1}=0$ and $a_{2}=0$, i.e., $Q=\frac{(2+d) z-(1+d)}{z^{2}}$ (and the proof is given in Appendix B).

First, consider the additional metrology delay of one run $(d=1)$ and the SSE for the benchmark are $a_{1}=0$ and $a_{2}=0$, i.e., $Q=\frac{3 z-2}{z^{2}}\left(\|Q\|_{\infty}=5\right)$. The Q-filter can guarantee the process output to be stable under the modeling uncertainty $\|\Delta\|_{\infty} \leq \frac{1}{5}$. To tolerate more modeling uncertainty, the contour plots for both the infinity norm of the second-order Q-filter with delay of one run and the SSE equation are plotted in Fig. 14, respectively.

In Fig. 14, for example, on the contour of $\|Q\|_{\infty}=3$ the optimal parameters of the second-order Q-filter are obtained as $a_{1}=-0.33$ and $a_{2}=0.065$ for $\|\Delta\|_{\infty} \leq \frac{1}{3}$. In addition, the minimum SSE $\sum e^{2}\left(a_{1}, a_{2}\right)$ for the benchmark $\left(P=P_{n}=1\right)$ is 5.363. It corresponds to the double EWMA controller with $\omega_{1}=0.935$ and $\omega_{2}=0.735$, the PCC controller with $\omega_{1}^{*}=$ $0.835 \pm 0.1944 i$ and $\omega_{2}^{*}=0.835 \mp 0.1944 i$. The simulations of the process outputs are shown in Fig. 15 where the nominal plant is set $P_{n}=1$, the actual plant is set $P=0.6667, P=1$ and $P=1.3333$, respectively, corresponding to $\|\Delta\|_{\infty} \leq \frac{1}{3}$, and the drift disturbance $(\delta=1)$ occurs after run 20 .

Similarly, one considers $d=2$ and the contours are plotted in Fig. 16. For example, on the contour of $\|Q\|_{\infty}=4$ the optimal parameters are obtained as $a_{1}=-0.35$ and $a_{2}=0.07$ under model uncertainty $\|\Delta\|_{\infty} \leq 0.25$.

It corresponds to the double EWMA controller with $\omega_{1}=$ 0.93 and $\omega_{2}=0.72$; the corresponding parameters of PCC controller are still complex numbers with $\omega_{1}^{*}=0.825 \pm 0.1984 i$ and $\omega_{2}^{*}=0.825 \mp 0.1984 i$. The simulations of the process outputs are illustrated in Fig. 17.

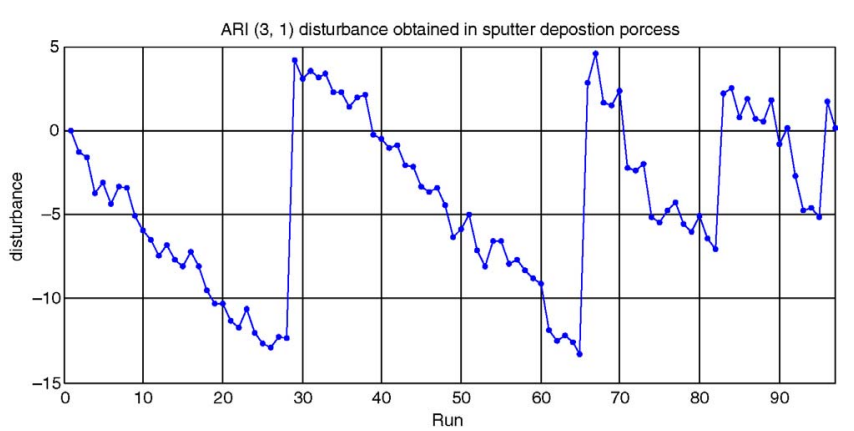

Fig. 18. $\operatorname{ARI}(3,1)$ disturbance obtained in sputter deposition process [27].

It is obvious that from Figs. 15 and 17 the presence of the metrology delay will result in poor performance and the optimal performance for the nominal plant under the robust stable condition is achieved by our proposed approach.

\section{NumericAl EXAMPLES With High ORDER Q-FILTER AND DISTURBANCES}

In this section, two examples with a third-order Q-filter are provided to show the performance improvement over EWMA, double EWMA, and PCC controllers. According to the internal model principle, the controlled output rejects disturbance without steady-state error if the reciprocal of the disturbance is modeled into the controller. Thus, higher order controllers will reject higher order disturbances. Because the proposed ODOB can be designed as a higher order controller, the performance improvements are expectable. 
TABLE I

COMPARISOns Among EWMA, Double EWMA, PCC AND ODOB Under AN ARI $(3,1)$ Disturbance

\begin{tabular}{|c|c|c|c|}
\hline Controller & Parameters & MSE & $\begin{array}{l}\text { Performance Improvement (\%) } \\
\frac{(8.3483-\mathrm{MSE})}{8.3483} \times 100 \%\end{array}$ \\
\hline EWMA & $\omega=0.79$ & 8.3483 & Benchmark \\
\hline Double EWMA & $\left\{\begin{array}{l}\omega_{1}=0.79 \\
\omega_{2}=-0.01\end{array}\right.$ & 8.2469 & $1.21 \%$ \\
\hline PCC & $\left\{\begin{array}{l}\omega_{1}^{*}=0.79 \\
\omega_{2}^{*}=-0.01\end{array}\right.$ & 8.2469 & $1.21 \%$ \\
\hline ODOB & $Q(z)=\frac{0.83 z^{2}-1.63 z+0.8}{z^{3}-1.95 z^{2}+0.93 z+0.02}$ & 8.0086 & $4.07 \%$ \\
\hline
\end{tabular}

TABLE II

Comparisons Among EWMA, Double EWMA, PCC AND ODOB Under the ARI(5, 1) DisturbanCE

\begin{tabular}{|l|c|c|c|}
\hline Controller & Parameters & MSE & $\begin{array}{c}\text { Performance Improvement }(\%) \\
\frac{(1.0204-\text { MSE })}{1.0204} \times 100 \%\end{array}$ \\
\hline EWMA & $\omega=1.05$ & 1.0204 & Benchmark \\
\hline Double EWMA & $\left\{\begin{array}{l}\omega_{1}=1 \\
\omega_{2}=0.04\end{array}\right.$ & 0.9855 & $3.42 \%$ \\
\hline PCC & $\left\{\begin{array}{l}\omega_{1}^{*}=1 \\
\omega_{2}^{*}=0.04\end{array}\right.$ & 0.9855 & $3.42 \%$ \\
\hline ODOB & $Q(z)=\frac{1.02 z^{2}-0.36 z-0.76}{z^{3}-0.39 z^{2}-0.54 z-0.17}$ & 0.8659 & $15.14 \%$ \\
\hline
\end{tabular}

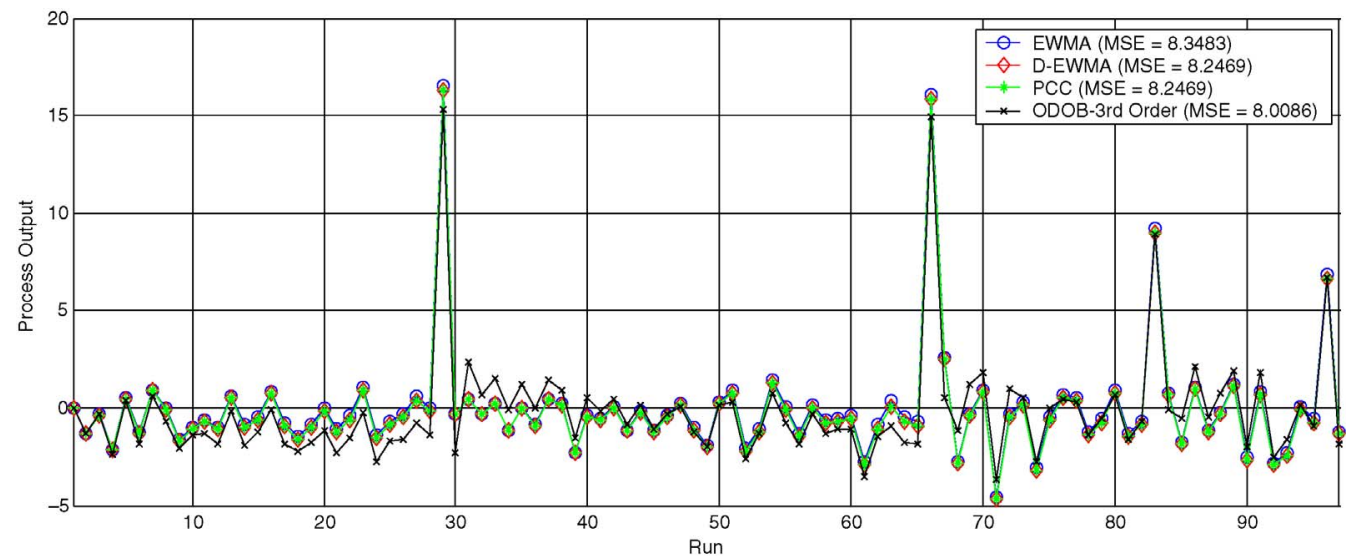

Fig. 19. Process outputs with the $\operatorname{ARI}(3,1)$ disturbance.

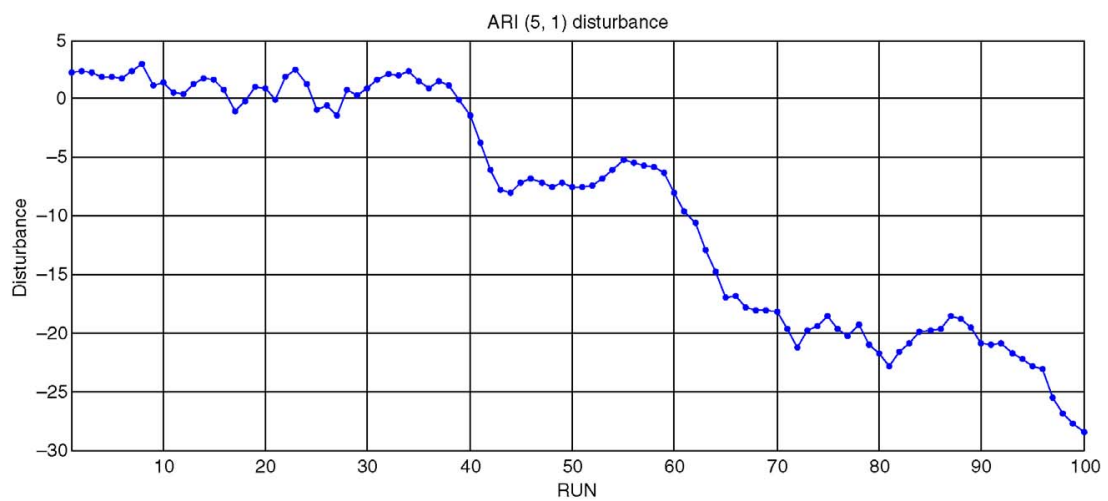

Fig. 20. $\operatorname{ARI}(5,1)$ anthropogenic disturbance data plot. 


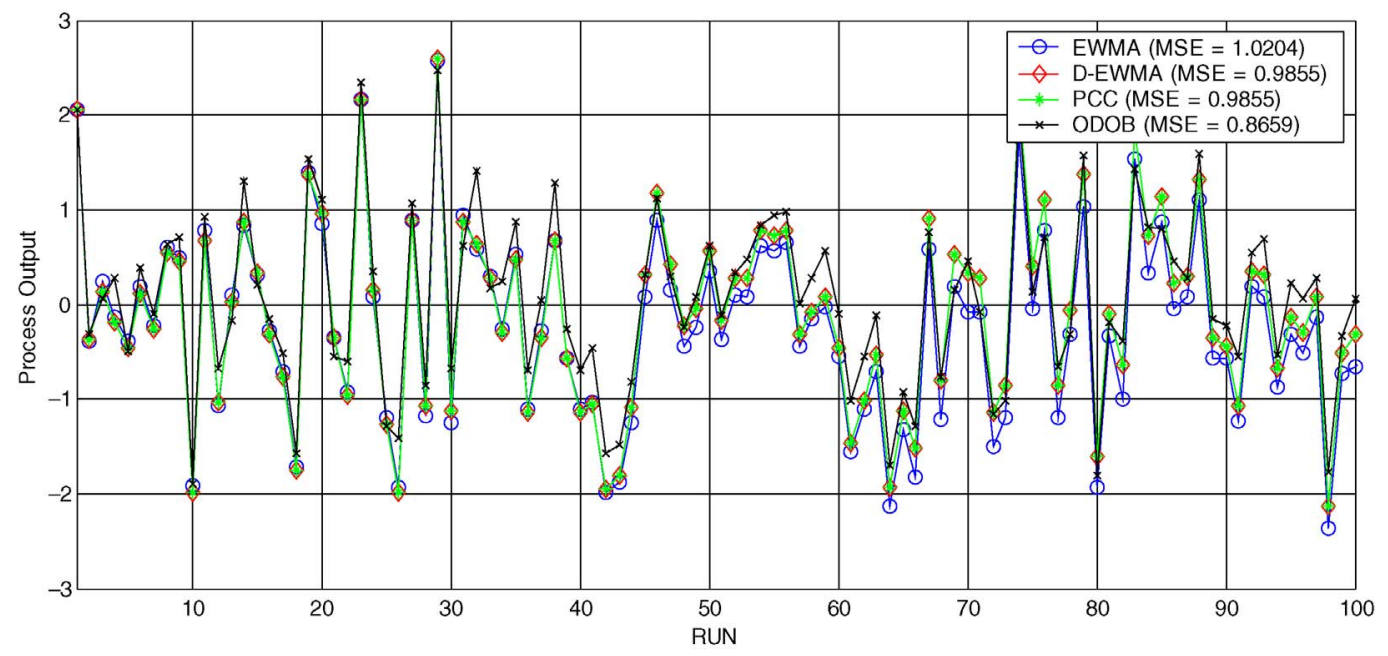

Fig. 21. Process outputs with an $\operatorname{ARI}(5,1)$ disturbance.

\section{A. An ARI $(3,1)$ Disturbance in Sputter Deposition Process}

$\operatorname{An} \operatorname{ARI}(3,1)$ disturbance is observed in the sputter deposition process [27] and the historical data series is plotted in Fig. 18. In this illustration, the actual plant is set as 1.2 and the nominal plant is $1(\xi=1.2)$ without metrology delay, and the reference target is 0 .

The best performance indexed by mean squared error (MSE) for EWMA, double EWMA, PCC, and ODOB can be obtained by exhaustive searching, and the performance results are shown in Fig. 19 and Table I.

If the EWMA is set as benchmark, the double EWMA and PCC both improve performance by $1.21 \%$ and the proposed ODOB up to $4.07 \%$.

\section{B. An ARI $(5,1)$ Anthropogenic Disturbance}

An $\operatorname{ARI}(5,1)$ anthropogenic disturbance is considered in the second example (Fig. 20). The actual plant is set as 1.2 and the nominal plant is $1(\xi=1.2)$ without metrology delay, and the reference target is 0 .

Following the same procedure as in the first example, the results were obtained and shown in Fig. 21 and Table II.

The performances of double EWMA, PCC, and the thirdorder ODOB over EWMA are improved by $3.42 \%, 3.42 \%$, and $15.14 \%$, respectively. These two examples demonstrate that higher order controllers can improve process performance when the process suffers from higher order disturbances.

\section{CONCLUSION}

In this paper, we provided a unified framework of the RtR controllers, ODOB scheme, in which the first-order Q-filter for the EWMA, the second-order Q-filter for the double EWMA and PCC controllers; moreover, the parameter set is larger than that of the PCC and is equivalent to the sets of EWMA and double EWMA. Based on this framework, we can enhance insight into the well-known established algorithms such as EWMA, double EWMA, and PCC controllers, and contribute to an improved understanding of how these algorithms operate and why they can be used successfully in practical application. Most importantly, the ODOB controller is more general than the conventional ones, i.e., higher order Q-filters can be designed to handle higher order disturbances, which the conventional ones can never do. Besides, we provided an effective augmented state-space method for obtaining stable region with/without finite metrology delays. Also, we provided a systematic method for obtaining the optimal parameters which guarantee the optimal performance for the nominal plant under the robust stability. When a process has larger metrology delays, following the approach of this paper to tune the parameters is considered to be appropriate. It is believed that this ODOB scheme can be further exploited to the adaptive control design field for more complicated perturbations.

\section{APPENDIX A}

\section{EWMA, DOUBLE EWMA, AND PCC CONTROLLERS}

Consider the process is a linear static model represented as

$$
y_{k}=\alpha+\beta \cdot u_{k}
$$

where $\alpha$ is the intercept term, $\beta$ is the process gain, $y_{k}$ is the process output, and $u_{k}$ is the process input obtained as

$$
u_{k}=\frac{T-\bar{a}_{k-1}}{b}
$$

where $T$ is the process target and $\bar{a}_{k-1}$ is estimated for the intercept term by the algorithm of the EWMA at $k-1$ run

$$
\bar{a}_{k-1}=\omega \cdot m_{k-1}+(1-\omega) \cdot \bar{a}_{k-2}
$$

where $m_{k}=y_{k}-b \cdot u_{k}, b$ is the off-line estimation of $\beta$ and $\omega$ is the EWMA weighting which is usually set between 0 and 1. The algorithm of the EWMA controller can be expressed as the discrete filter. One can take z-transform in (A3), so the EWMA filter merged with a unit delay is represented as

$$
\begin{aligned}
\bar{a}_{k-1} & =\omega \cdot m_{k-1}+(1-\omega) \cdot \bar{a}_{k-2} \\
& \Leftrightarrow \bar{A}_{k} \cdot z^{-1}=\omega \cdot M \cdot z^{-1}+(1-\omega) \cdot \bar{A}_{k} \cdot z^{-2} \\
& \Leftrightarrow \bar{A}_{k} \cdot z=\omega \cdot M \cdot z+(1-\omega) \cdot \bar{A}_{k} \\
& \Leftrightarrow F_{E}(z) \cdot z^{-1}=\frac{\bar{A}_{k}(z)}{M(z)} \cdot z^{-1}=\frac{\omega}{z-(1-\omega)} .
\end{aligned}
$$


The double EWMA and the PCC controllers are applied to the process model

$$
y_{k}=\alpha+\beta \cdot u_{k}+\delta \cdot k
$$

where $\delta$ is the average drift per run. The double EWMA algorithm is represented as

$$
\begin{gathered}
r_{k}=\omega_{1} m_{k}+\left(1-\omega_{1}\right)\left(r_{k-1}+p_{k-1}\right) \\
p_{k}=\omega_{2}\left(m_{k}-r_{k-1}\right)+\left(1-\omega_{2}\right) p_{k-1} \\
\bar{a}_{k}=r_{k}+p_{k}
\end{gathered}
$$

where $r_{k}$ and $p_{k}$ are the one-step-ahead prediction for $\alpha$ and $\delta k, \omega_{1}$ and $\omega_{2}$ are the weightings which weight noise and drift, respectively. The recipe for the $k$ run is $u_{k}=\frac{T-\bar{a}_{k-1}}{b}$. The algorithm of the double EWMA controller can be expressed as the discrete filter. The double EWMA filter merged with a unit delay is represented as

$$
F_{D}(z) \cdot z^{-1}=\frac{\left(\omega_{1}+\omega_{2}\right) z-\omega_{1}}{z^{2}-\left(2-\omega_{1}-\omega_{2}\right) z+\left(1-\omega_{1}\right)} .
$$

The PCC algorithm is similar to the double EWMA algorithm that is represented as

$$
\begin{gathered}
r_{k}=\omega_{1}^{*} m_{k}+\left(1-\omega_{1}^{*}\right) r_{k-1} \\
p_{k}=\omega_{2}^{*}\left(m_{k}-r_{k-1}\right)+\left(1-\omega_{2}^{*}\right) p_{k-1} \\
\bar{a}_{k}=r_{k}+p_{k}
\end{gathered}
$$

where $\omega_{1}^{*}$ and $\omega_{2}^{*}$ are the weightings which weight noise and drift, respectively. $r_{k}$ and $p_{k}$ are the estimation of parameters. The PCC filter merged with a unit delay can be represented as

$$
F_{P}(z) \cdot z^{-1}=\frac{\left(\omega_{1}^{*}+\omega_{2}^{*}\right) z-\left(\omega_{1}^{*}+\omega_{2}^{*}-\omega_{1}^{*} \omega_{2}^{*}\right)}{z^{2}-\left(2-\omega_{1}^{*}-\omega_{2}^{*}\right) z+\left(1-\omega_{1}^{*}\right)\left(1-\omega_{2}^{*}\right)} .
$$

The double EWMA and the PCC controller can be equivalently represented in the ODOB structure. According to the Jury's test, the stable region of the double EWMA controller is obtained as

$$
0<\xi<\frac{4}{2 \omega_{1}+\omega_{2}}
$$

and the stable region of the PCC controller is obtained as

$$
0<\xi<\frac{4}{2\left(\omega_{1}^{*}+\omega_{2}^{*}\right)-\omega_{1}^{*} \omega_{2}^{*}} .
$$

Del Castillo et al. discussed the stable conditions for the SISO and MIMO PCC controller. But, the stable condition has little imperfections. An example is given to demonstrate the result in (A15) is correct. If the weightings of the PCC controller are set $\omega_{1}^{*}=0.3$ and $\omega_{2}^{*}=0.4$, the stable region of the PCC controller is $0<\xi<3.125$. However, the overall stable region of the PCC controller obtained in Del Castillo et al. is $0<\xi<2.857$.

\section{APPENDIX B}

\section{DERIVATION OF LEMMA 1}

When the additional metrology delay is considered, the error equation between the process target and output without considering the measurement noise is obtained as

$$
\begin{aligned}
E(z)=Y(z)-T(z)= & {\left[\frac{(\xi-1)\left(z^{d}-Q\right)}{z^{d}+(\xi-1) Q}\right] \cdot T(z) } \\
& +\left[\frac{\left(z^{d}-Q\right)}{z^{d}+(\xi-1) Q}\right] \cdot H_{o}(z) .
\end{aligned}
$$

In (B1), the process target $T$ has been set to zero without loss of generality. The performance for the condition with $P=P_{n}$ is used as the benchmark, and the second-order Qfilter designed in (9) with the constraint of (28) is substituted into (B1). Take the z-transform in (B1) where $H_{o}(z)=\frac{\delta z}{(z-1)^{2}}$ (drift disturbance) and the error equation can be represented as follows:

$$
\begin{aligned}
& z^{1-d} \delta\left[\left(1-2 z-a_{1} z-a_{2}\right)+z^{d}\left(z^{2}+a_{1} z+a_{2}\right)\right. \\
& E(z)=\frac{\left.+d(1-z)\left(1+a_{1}+a_{2}\right)\right]}{(z-1)^{2}\left(z^{2}+a_{1} z+a_{2}\right)} .
\end{aligned}
$$

According to Parseval's theorem [25], the SSE equation with metrology delay can be derived from (B2). The optimal parameters of the second-order Q-filter with metrology delay for the performance of the benchmark are obtained for $a_{1}=0$ and $a_{2}=0$, i.e., $Q=\frac{(2+d) z-(1+d)}{z^{2}}$.

\section{REFERENCES}

[1] A. Ingolfsson and E. Sachs, "Stability and sensitivity of an EWMA controller," J. Quality Technol., vol, 25, no. 4, pp. 271-287, Oct. 1993.

[2] S. W. Bulter and J. A. Stefani, "Supervisory run-to-run control of polysilicon gate etch using in situ ellipsometry," IEEE Trans. Semiconductor Manuf., vol. 7, no. 2, pp. 193-201, May 1994.

[3] E. Sachs, A. Hu, and A. Ingolfsson, "Run by run process control: Combining SPC and feedback control," IEEE Trans. Semiconductor Manuf., vol. 8, no. 1, pp. 26-43, Feb. 1995.

[4] D. S. Boning, W. P. Moyne, T. H. Smith, J. Moyne, R. Telfeyan, A. Hurwitz, S. Shellman, and J. Taylor, "Run by run control of chemical mechanical polishing," IEEE Trans. Compon. Packag. Manuf. Technol., Part C, vol. 19, no. 4, pp. 307-314, Oct. 1996.

[5] X. A. Wang and R. L. Mahajan, "Artificial neural network model-based run-to-run process controller," IEEE Trans. Compon. Packag. Manuf. Technol., Part C, vol. 19, no. 1, pp. 19-26, Jan. 1996.

[6] E. Del Castillo and J. Y. Yeh, "An adaptive run-to-run optimizing controller for linear and nonlinear semiconductor processes," IEEE Trans. Semiconductor Manuf., vol. 11, no. 2, pp. 285-277, May 1998.

[7] T. H. Smith, D. S. Boning, J. Stefani, and S. W. Bulter, "Run by run advanced process control of metal sputter deposition," IEEE Trans. Semiconductor Manuf., vol. 11, no. 2, pp. 276-284, May 1998.

[8] E. S. Hamby, P. T. Kabamba, and P. P. Khargonekar, "A probabilistic approach to run-to-run control," IEEE Trans. Semiconductor Manuf., vol. 11, no. 4, pp. 654-669, Nov. 1998.

[9] E. Del Castillo, "Long run and transient analysis of a double EWMA feedback controller," IIE Trans., vol. 31, no. 12, pp. 1157-1169, Sep. 1999.

[10] S. Adivikolanu and E. Zafiriou, "Extensions and performance/robustness tradeoffs of the EWMA run-to-run controller by using the internal model control structure," IEEE Trans. Electron. Packag. Manuf., vol. 23, no. 1, pp. 56-68, Jan. 2000.

[11] A. Chen and R. S. Guo, "Age-based double EWMA controller and its application to CMP processes," IEEE Trans. Semiconductor Manuf., vol. 14, no. 1, pp. 11-19, Feb. 2001. 
[12] S. T. Tseng, R. J. Chou, and S. P. Lee, "A study on a multivariate EWMA controller," IIE Trans., vol. 34, no. 6, pp. 541-549, Jun. 2002.

[13] J. Wang, Q. P. He, S. J. Qin, C. A. Bode, and M. A. Purdy, "Recursive least squares estimation for run-to-run control with metrology delay and its application to STI etch process," IEEE Trans. Semiconductor Manuf., vol. 18, no. 2, pp. 309-319, May 2005.

[14] R. P. Good and S. J. Qin, "On the stability of MIMO EWMA run-to-run controllers with metrology delay," IEEE Trans. Semiconductor Manuf., vol. 19, no. 1, pp. 78-86, Feb. 2006.

[15] M. F. Wu, C. H. Lin, D. S. H. Wong, S. S. Jang, and S. T. Tseng, "Performance analysis of EWMA controllers subject to metrology delay," IEEE Trans. Semiconductor Manuf., vol. 21, no. 3, pp. 413-425, Aug. 2008.

[16] E. D. Castillo, "Some properties of EWMA feedback quality adjustment schemes for drifting disturbances," J. Quality Technol., vol. 33, no. 2, pp. 153-166, 2001.

[17] G. E. P. Box and G. M. Jenkins, Time Series Analysis-Forecasting and Control. San Francisco, CA: Holden-Day, 1976.

[18] K. Ohishi, M. Nakao, K. Ohnishi, and K. Miyachi, "Microprocessorcontrolled DC motor for load-in-sensitive position servo system," IEEE Trans. Ind. Electron., vol. 34, no. 1, pp. 44-49, Feb. 1987.

[19] T. Umeno and Y. Hori, "Robust speed control of DC servomotors using modern two degrees-of-freedom controller design," IEEE Trans. Ind. Electron., vol. 38, no. 5, pp. 363-368, Oct. 1991.

[20] M. Green and D. Limebeer, Linear Robust Control. Upper Saddle River, NJ: Prentice-Hall, 1994

[21] E. I. Jury, Theory and Application of the Z-Transform Method. Malabar, FL: Krieger, 1973.

[22] R. P. Good and S. J. Qin, "On the stability of MIMO EWMA run-to-run controllers with metrology delay," IEEE Trans. Semiconductor Manuf., vol. 19, no. 1, pp. 78-86, Feb. 2006.

[23] J. V. Beck and K. J. Arnold, Parameter Estimation in Engineering and Science. New York: Wiley, 1977.

[24] K. Zhou and J. C. Doyle, Essentials of Robust Control. Upper Saddle River, NJ: Prentice-Hall, 1998.

[25] C. L. Philips and H. T. Nagle, Digital Control System Analysis and Design. Upper Saddle River, NJ: Prentice-Hall, 1995.

[26] J. S. Arora, Introduction to Optimum Design. New York: McGraw-Hill, 1989.

[27] J. H. Chen, T. W. Kuo, and A. C. Lee, "Run-by-run process control of metal sputter deposition: Combining time series and extended Kalman filter," IEEE Trans. Semiconductor Manuf., vol. 20, no. 3, pp. 272-285, Aug. 2007.

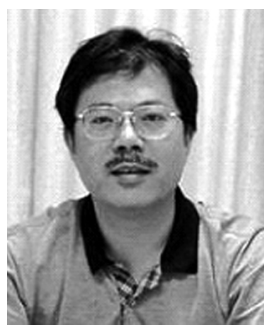

An-Chen Lee received the B.S. and M.S. degrees in power mechanical engineering from National TsingHua University, Hsinchu, Taiwan, and the Ph.D. degree in mechanical engineering from the University of Wisconsin-Madison, Madison, in 1986.

$\mathrm{He}$ is a Chair Professor with National Chiao Tung University, Hsinchu, and is currently a Professor with the Department of Mechanical Engineering. His current research interests include computer numerical control machine tool control technology, magnetic bearing technology, rotor dynamics and control, and semiconductor manufacturing process control.

Dr. Lee has served as an editorial board member of the International Journal of Precision Engineering and Manufacturing, the Chinese Society of Mechanical Engineers, and the International Journal of Applied Mechanics and Engineering. He was the recipient of the National Science Committee (NSC) Excellent Research Award from 1991 to 1992, the NSC Distinguished Research Award from 1993 to 1994, 1995 and 1996, 1997 to 1998, the NSC Research Fellow from 1999 to 2001, and 2002 to 2004, the NSC Research Fellowship Award in 2005, the Chinese Society of Mechanical Engineers Distinguished Engineering Professor Award in 1995, and the Chinese Society of Mechanical Engineers Distinguished Engineering Professor Award in 2001

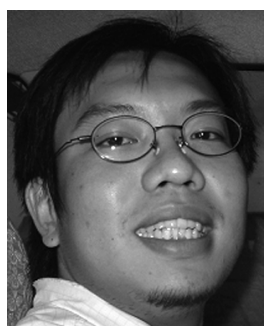

Yi-Ren Pan was born in Pingtung, Taiwan, in 1978. He received the B.S. and M.S. degrees in mechanical engineering from National Chiao Tung University, Hsinchu, Taiwan, in 2001 and 2003, respectively. $\mathrm{He}$ is currently working toward the Ph.D. degree in mechanical engineering from the Department of Mechanical Engineering, National Chiao Tung University.

His current research interests include run-to-run control, process control, and robust control.

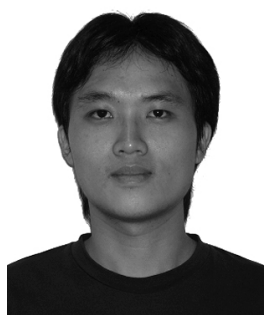

Ming-Tsung Hsieh was born in Taichung, Taiwan, in 1982. He received the B.S. and M.S. degrees in mechanical engineering from National Central University, Taoyuan, Taiwan, and National Chiao Tung University, Hsinchu, Taiwan, in 2005 and 2007 , respectively.

He is currently with the Department of Mechanical Engineering, National Chiao Tung University. His current research interests include run-to-run control and process control. 